\title{
Connections of the mouse subfornical region of the lateral hypothalamus (LHsf)
}

\author{
Müzeyyen Ugur ${ }^{1,2,4,5}$, Stéphane Doridot ${ }^{3}$, Susanne E la Fleur ${ }^{4,5}$, Pierre Veinante*1,2, \\ Dominique Massotte*1
}

${ }^{1}$ Institut des Neurosciences Cellulaires et Intégratives, Centre National de la Recherche Scientifique, Strasbourg, France.

${ }^{2}$ Université de Strasbourg, Strasbourg, France.

${ }^{3}$ Chronobiotron, Centre National de la Recherche Scientifique, Strasbourg, France

${ }^{4}$ Amsterdam UMC, University of Amsterdam, Department of Endocrinology and

Metabolism, Laboratory of Endocrinology, Department of Clinical Chemistry, Amsterdam

Neuroscience, Amsterdam, Netherlands.

${ }^{5}$ Metabolism and Reward Group, Netherlands Institute for Neuroscience, Royal Netherlands Academy of Arts and Sciences (KNAW), Amsterdam, Netherlands.

\section{*Corresponding authors:}

\section{Dominique Massotte}

\section{Pierre Veinante}

Institut des Neurosciences Cellulaires et Intégratives, CNRS UPR3212, 8 Allée du Général Rouvillois, 67000, Strasbourg, France

DM: +33 388456 652; d.massotte@unistra.fr

PV: +33 388456 609; veinantep@inci-cnrs.unistra.fr

\begin{abstract}
The lateral hypothalamus is a major integrative hub with a complex architecture characterized by intricate and overlapping cellular populations expressing a large variety of neuromediators. In rats, the subfornical lateral hypothalamus (LHsf) was identified as a discrete area with very specific outputs, receiving a strong input from the nucleus incertus, and involved in defensive and foraging behaviors. We identified in the mouse lateral hypothalamus a discrete subfornical region where a conspicuous cluster of neurons express the mu opioid receptor. We thus examined the inputs and outputs of this LHsf region in mice
\end{abstract}


using retrograde tracing with the cholera toxin B subunit and anterograde tracing with biotin dextran amine, respectively. We identified a connectivity profile largely similar, although not identical, to what has been described in rats. Indeed, the mouse LHsf has strong reciprocal connections with the lateral septum, the ventromedial hypothalamic nucleus and the dorsal premamillary nucleus, in addition to a dense output to the lateral habenula. However, the light input from the nucleus incertus and the moderate bidirectional connectivity with nucleus accumbens are specific to the mouse LHsf. A preliminary neurochemical study showed that LHsf neurons expressing mu opioid receptors also coexpress calcitonin gene-related peptide or somatostatin and that the reciprocal connection between the LHsf and the lateral septum may be functionally modulated by enkephalins acting on mu opioid receptors. These results suggest that the mouse LHsf may be hodologically and functionally comparable to its rat counterpart, but more atypical connections also suggest a role in consummatory behaviors.

Key words:

mu opioid receptor, anterograde tracing, retrograde tracing, defensive behavior, feeding 


\section{Abbreviations}

$3 \mathrm{~N}$ : oculomotor $\mathrm{N}$

$3 \mathrm{~V}: 3^{\text {rd }}$ ventricle

A24a: anterior cingulate area $24 \mathrm{a}$

A24ba: anterior cingulate area $24 b$

A25: anterior cingulate area 25

A32: anterior cingulate area 32

AAA: anterior amygdaloid area

an: anterior commissure

aca: anterior commissure, anterior limb

Acb: $\mathrm{N}$ accumbens

AcbC: $\mathrm{N}$ accumbens, core

AcbSh: $\mathrm{N}$ accumbens, shell

ACo: anterior cortical amygdaloid $\mathrm{N}$

$\mathrm{AD}$ : anterodorsal thalamic $\mathrm{N}$

$\mathrm{AH}$ : anterior hypothalamic area

AHiA: amygdalohippocampal area, anterior part

AI: agranular insular cortex

AOM: anterior olfactory $\mathrm{N}$, medial part

Aq: cerebral aqueduct

Arc :arcuate hypothalamic $\mathrm{N}$

ATg: anterior tegmental $\mathrm{N}$

Bar: Barrington's N

BDA: Biotin dextran amine

BLA: basolateral amygdaloid $\mathrm{N}$, anterior

BLP: basolateral amygdaloid $\mathrm{N}$, posterior

BMA: basomedial amygdaloid $\mathrm{N}$, anterior

BMP: basomedial amygdaloid $\mathrm{N}$, posterior

BST: bed $\mathrm{N}$ of the stria terminalis

BSTIA: BST, intraamygdlaoid

BSTLD: lateral BST, dorsal

BSTLP: lateral BST, posterior

BSTLV: lateral BST, ventral

BSTMA: medial BST, anterior part

BSTMP: medial BST, posterior

BSTMPI: medial BST, posterointermediate

BSTMPL: medial BST, posterolateral

BSTMPM: medial BST, posteromedial part

BSTMV: medial BST, ventral

cb: cerebellum cc: corpus callosum

CeC: Central amygdaloid N, capsular

CeL: central amygdaloid $\mathrm{N}$, lateral

CeM: central amygdaloid $\mathrm{N}$, medial

CGA: central gray, alpha part

CGB: central gray, beta part

CGRP: calcitonin gene related peptide

$\mathrm{CM}$ : central medial thalamic $\mathrm{N}$

CnF: cuneiform $\mathrm{N}$

cp: cerebral peduncle

$\mathrm{CPu}$ : caudate putamen

CTB: cholera toxin B subunit

DEn: dorsal endopiriform $\mathrm{N}$

DG: Dentate gyrus

Dk: $\mathrm{N}$ of Darkschewitsch

DLL: dorsal $\mathrm{N}$ of the lateral lemniscus

dlPAG: dorsolateral periaqueductal gray

DM: dorsomedial hypothalamic $\mathrm{N}$

dmPAG: dorsomedial periaqueductal gray

DR: dorsal raphe $\mathrm{N}$

DRC: dorsal raphe $\mathrm{N}$, caudal

DRD: dorsal raphe $\mathrm{N}$, dorsal

DRL: dorsal raphe $\mathrm{N}$, lateral

DRV: dorsal raphe $\mathrm{N}$, ventral

DTg: dorsal tegmental N

DTT: dorsal tenia tecta

EA: extended amygdala

f: fornix

fmi: forceps minor of the corpus callosum

fr: fasciculus retroflexus

hbc: habenular commissure

HDB: $\mathrm{N}$ of the horizontal limb of the diagonal band

I: intercalated nuclei of the amygdala

Im: intercalated nucleus of the amygdala, main part

ic: internal capsule

IC: inferior colliculus

ICjM: island of Calleja, major island

IF: interfascicular $\mathrm{N}$

IPN: interpeduncular $\mathrm{N}$ 
IsRt: isthmic reticular formation

JPLH: juxtaparaventricular lateral hypothalamus

La: lateral amygdaloid $\mathrm{N}$

LaDL: Lateral amygdaloid N, dorsolateral part

LaV: Lateral amygdaloid N, ventral part

LA: lateroanterior hypothalamic $\mathrm{N}$

LC: locus coeruleus

LDTg: laterodorsal tegmental $\mathrm{N}$

LH: lateral hypothalamic area

LHAa: lateral hypothalamic area, anterior region (Rat)

LHAd: lateral hypothalamic area, dorsal region (Rat)

LHAjv: lateral hypothalamic area, juxtaventromedial region (Rat)

LHAsfa: lateral hypothalamic area, subfornical region, anterior zone (Rat)

LHAsfp: lateral hypothalamic area, subfornical region, posterior zone (Rat)

LHb: lateral habenular $\mathrm{N}$

LHbL: lateral habenular $\mathrm{N}$, lateral part

LHbM: lateral habenular N, medial part

LHsf: lateral hypothalamus, subfornical region (mouse)

11: lateral lemniscus

IPAG: lateral periaqueductal gray

LPBC: lateral parabrachial $\mathrm{N}$, central

LPBD: lateral parabrachial N, dorsal

LPBE: lateral parabrachial $\mathrm{N}$, external

LPBI: lateral parabrachial $\mathrm{N}$, internal

LPBS: lateral parabrachial N, superior

LPBV: lateral parabrachial $\mathrm{N}$, ventral

LPO: lateral preoptic area

LS: lateral septum

LSD: lateral septal $\mathrm{N}$, dorsal

LSI: lateral septal $\mathrm{N}$, intermediate

LSV: lateral septal $\mathrm{N}$, ventral

LV: lateral ventricle

MA3: medial accessory oculomotor $\mathrm{N}$

MD: mediodorsal thalamic $\mathrm{N}$
MeA: medial amygdaloid $\mathrm{N}$

Me5: mesencephalic trigeminal $\mathrm{N}$ and tract

MeAD: medial amygdaloid $\mathrm{N}$, anterodorsal

MeAV: medial amygdaloid N, anteroventral

MePD: medial amygdaloid N, posterodorsal

MePV: medial amygdaloid N, posteroventral

MHb: medial habenular $\mathrm{N}$

MiTg: microcellular tegmental N

$\mathrm{ml}$ : medial lemniscus

mlf: medial longitudinal fasciculus

ML: medial mamillary N, lateral

MM: medial mamillary $\mathrm{N}$, medial

MnPO: median preoptic $\mathrm{N}$

MnR: median raphe $\mathrm{N}$

MO: medial orbital cortex

MPA: medial preoptic area

MPB: medial parabrachialN

MPL: medial paralemniscal $\mathrm{N}$

MPO: medial preoptic $\mathrm{N}$

$\mathrm{mRt}$ : mesencephalic reticular formation

MS: medial septal N

mt: mamillothalamic tract

MT: medial terminal $\mathrm{N}$

MTu: medial tuberal hypothalamic $\mathrm{N}$

$\mathrm{N}$ : nucleus

NI: N Incertus

ot: optic tract

$\mathrm{Pa}$ : paraventricular hypothalamic $\mathrm{N}$

PAG: periaqueductal gray

PBP: parabrachial pigmented $\mathrm{N}$ of the VTA

pc: posterior commissure

PDR: posterodorsal raphe $\mathrm{N}$

PH: posterior hypothalamic $\mathrm{N}$

PIF: parainterfascicular N of the VTA

PIL: posterior intralaminar thalamic $\mathrm{N}$

Pir: piriform cortex

PLCo: posterolateral cortical amygdaloid area pm: principal mamillary tract

PMCo: posteromedial cortical amygdaloid area

PMD: premamillary $\mathrm{N}$, dorsal part 
PMnR: paramedian raphe $\mathrm{N}$

PMV: premamillary $\mathrm{N}$, ventral part

PN: paranigral $\mathrm{N}$ of the VTA

PoT: posterior thalamic nuclear group, triangular

PR: prerubral field

PrC: precommissural $\mathrm{N}$

PT: Paratenial thalamic N

PTg: pedunculotegmental N

PV: paraventricular thalamic $\mathrm{N}$

PVA: paraventricular thalamic $\mathrm{N}$, anterior

$\mathrm{RCh}$ : retrochiasmatic area

RChL: retrochiasmatic area, lateral

Re: reuniens thalamic $\mathrm{N}$

$\mathrm{RLi}$ : rostral linear $\mathrm{N}$

RM: retromamillary $\mathrm{N}$

RMC: red N, magnocellular part

RMM: retromammillary $\mathrm{N}$, medial

rPAG: rostral periaqueductal gray

RPC: red N, parvicellular part

Rt: reticular thalamic $\mathrm{N}$

SCh: suprachiasmatic N

scp: superior cerebellar peduncle

Shi: septohippocampal N

Shy: septohypothalamic N

sm: stria medullaris

SNC: substantia nigra, compact part

SNR: substantia nigra, reticular part
SO: supraoptic N

sox: supraoptic decussation

SST: somatostatin

st: stria terminalis

StHy: striohypothalamic N

Su3: supraoculomotor periaqueductal gray

Su3C: supraoculomotor cap

Sub: submedius thalamic $\mathrm{N}$

VDB: $\mathrm{N}$ of the vertical limb of the diagonal band vlPAG: ventrolateral periaqueductal gray

VMH: ventromedial hypothalamic $\mathrm{N}$

VMHC: ventromedial hypothalamic $\mathrm{N}$, central part

VMHDM: ventromedial hypothalamic N,

dorsomedial part

VMHVL: ventromedial hypothalamic $\mathrm{N}$,

ventrolateral part

VMPO: ventromedial preoptic $\mathrm{N}$

VO: ventral orbital cortex

VP: ventral pallidum

vsc: ventral spinocerebellar tract

VTA: ventral tegmental area

VTAR: ventral tegmental area, rostral part

VTg: ventral tegmental $\mathrm{N}$

xscp: decussation of the superior cerebellar

peduncle

ZI: zona incerta 


\section{INTRODUCTION}

The lateral hypothalamus (LH) represents a major integrative hub of central and peripheral information and, accordingly, controls a large area of adaptive functions. It is involved in the regulation of sleep-wake states, defense and arousal, reproduction, ingestive behavior and energy balance but is also a key player in motivated behaviors related to the rewarding aspects of food (Berthoud and Munzberg 2011). This region presents a complex architecture and expresses a large variety of neuromediators with often intricate and overlapping phenotypic patterns (Bonnavion et al. 2016). The opioid system critically modulates the activity of the LH with mu opioid receptors mainly expressed in the orexinergic perifornical area (Georgescu et al. 2003; Ardianto et al. 2016). Interestingly, a population of large neurons expressing mu opioid receptors has been identified in the subfornical area (LHsf) of knock-in mice expressing the receptor in fusion with the red fluorescent protein mCherry (Erbs et al. 2015). A role in the modulation of defensive and foraging behaviors depending on motivational and emotional states was also suggested for this area (Goto et al. 2001). In rats, the LHsf indeed receives various inputs including afferents from the parabrachial nucleus (Bester et al. 1997), the central gray/nucleus incertus (Goto et al. 2001), the lateral septum (LS) (Risold and Swanson 1997), and the periaqueductal gray (Goto et al. 2005). The LHsf also projects to multiple regions including other hypothalamic areas, the lateral habenula, the septal nuclei and the amygdala (Goto et al. 2005). Recently, Reppuci and Petrovich showed increased c-fos activation in the LHsf of both male and female rats upon food access (Reppucci and Petrovich 2018). Indeed, the LHsf in rats is also reciprocally connected to the shell of the nucleus accumbens (AcbSh), either directly (from the dorsomedial part or hedonic hotspot) or via the LS or the ventral pallidum (Thompson and Swanson 2010; Zahm et al. 2013; Urstadt and Stanley 2015). In addition, lesion of the anterior AcbSh increases cfos expression in the LHsf (Baldo et al. 2004) suggesting that the closed AcbSh-LHsf loop may modulate food motivation.

Although the LHsf is very likely a key player in the regulation of motivational aspects related to feeding behavior, the connectivity of this region has not been described in mouse so far. Indeed, while several studies addressed the connectivity of LH subregions in rats (Goto et al. 2005; Hahn and Swanson 2010, 2015), it is not the case for mice and the parcellation of the LH remains to be defined in this species. We therefore mapped the afferents to the LHsf, defined by the concentration of mu opioid receptor-expressing neurons, using retrograde tracing with the cholera toxin B subunit (CTB) and identified the output areas using 
anterograde tracing with biotinylated dextran amine (BDA). In addition, we also obtained preliminary results concerning the neurochemical profile of the neurons in this area.

\section{MATERIALS AND METHODS}

\section{Animals}

Male $(\mathrm{N}=7)$ and female $(\mathrm{N}=15)$ Mu-mCherry knock-in mice expressing the mu opioid receptor fused at its C-terminus to the red fluorescent protein mCherry (Erbs et al. 2015) aged 10-24 weeks, (20-40g) were used. The genetic background of the mice is C57BL6/J:129SvPas (50:50) mice. Mice were housed under standard conditions $\left(21 \pm 1^{\circ} \mathrm{C}\right.$, $55 \pm 10 \%$ humidity) on a $12 \mathrm{~h}$ dark-light cycle with food and water ad libitum. All experiments were approved by the Comité d'éthique en Matière d'expérimentation animale (authorization number 2015304113547b (APAFIS \#300.02) and conducted in agreement with the European Communities Council Directive 2010/63/EU for animal experiments.

\section{Retrograde and Anterograde Tracing}

Mice were anesthetized with (i.p., $5 \mathrm{~mL} / \mathrm{kg})$ injection of ketamine $(87 \mathrm{mg} / \mathrm{kg})$ and xylazine $(13 \mathrm{mg} / \mathrm{kg}) .0 .1 \mathrm{~mL}$ of local anesthetic $1 \%$ lidocaïne was subcutaneously injected under the scalp before placing the animal in the stereotaxic frame. A hydrating gel (Ocryl-gel) was regularly applied on the eyes of the animal to avoid dehydration. An incision was made in the scalp and a unilateral hole was drilled in the skull at the appropriate coordinates determined using the $4^{\text {th }}$ edition of the Mouse Brain Atlas (Paxinos and Franklin 2012) are listed in table 1. A glass micropipette (20-30 $\mu \mathrm{m}$ tip diameter) containing the retrograde tracer cholera toxin subunit B (CTB; $0.25 \%$ in $0.1 \mathrm{M}$ Tris and $0.1 \% \mathrm{NaCl}$; Sigma, France Cat:C9903) or the anterograde tracer biotin dextran amine (BDA) at $2 \%$ in $0.9 \% \mathrm{NaCl}(10,000 \mathrm{MW})$ (Molecular Probes D1956) was lowered into the target area. The tracer was ejected by iontophoresis using constant current source (Midgard; $3 \mu \mathrm{A}, 7 \mathrm{sec}$ on/off intervals) during $8 \pm 1$ min for CTB or during $12 \pm 1 \mathrm{~min}$ for BDA. After microinjection, the pipette was left in place for $10 \mathrm{~min}$ and then removed slowly. The incision was closed with two fastenings and the animal was left in a clean cage under a warming lamp until awakening. It was then transferred to the home cage in the animal facility under daily monitoring with Metacam ( $2 \mathrm{mg} / \mathrm{kg}$ s.c.) injected daily for 3 days.

\section{Tissue preparation}

Tracers revelation 
Eight to ten days post-surgery, mice were anesthetized with Dolethal $(200 \mathrm{mg} / \mathrm{L} ; 100 \mu \mathrm{L} / 10 \mathrm{~g}$ i.p.) and perfused intracardiacally with $100 \mathrm{ml}$ of $4 \%$ paraformaldehyde (Sigma, St Louis, MO, USA) (at 2-4 ${ }^{\circ} \mathrm{C}$ ) in phosphate buffer (PB 0.1 M, pH 7.4). Brains were post-fixed for 24 hours at $4^{\circ} \mathrm{C}$ in $4 \%$ PFA solution, washed with $0.1 \mathrm{M} \mathrm{PB} \mathrm{pH} 7.4$ and embedded in $4 \%$ agar. Coronal sections $(40 \mu \mathrm{m})$ were obtained with a Vibratome (VT 1000S, Leica, Deerfield, IL) and were serially collected in PB. Sections were kept at $4^{\circ} \mathrm{C}$ until further processing.

Detection by immunofluorescence was performed according to standard protocols. Briefly, floating sections were incubated in the blocking solution (PB $0.1 \mathrm{M} \mathrm{pH} \mathrm{7.4,0.2 \%} \mathrm{Tween} 20$ (Sigma, St Louis, MO, USA), 5\% normal donkey or goat serum depending on the secondary antibody) for 1 hour at room temperature (RT). Sections were the incubated with goat antiCTB (List Biological Laboratories Cat \#703, 1:10,000) or AlexaFluor 488 conjugated streptavidin (Molecular Probes, S32354, 1:500) diluted in PBST (PB 0.1 M pH 7.4, 0.2\% Tween 20 overnight at $4^{\circ} \mathrm{C}$. Sections were washed three times with PBST and incubated for 2 hours at RT with AlexaFluor-conjugated donkey anti-goat antibodies (AlexaFluor 488Molecular probes A-11055, 1:500, AlexaFluor 647- Molecular probes A-21447). Sections were washed three times with PBST, one time with PB and mounted on SuperfrostTM glass (Menzel-Glaser) with Mowiol (Calbiochem, Darmstadt, Germany) and 4', 6-diamidino-2phenylindole (DAPI) (Roche Diagnostic, Mannheim, Germany) $(0.5 \mu \mathrm{g} / \mathrm{ml})$.

\section{Colocalization with neuronal markers}

Calcitonin gene-related peptide (CGRP), somatostatin (SST) and methionine-enkephalin (met-enk) immunoreactivities were respectively detected using sheep polyclonal anti-CGRP (Abcam Ab22560, 1:2000), rat monoclonal anti-SST (Millipore MAB354, 1:1000) and rabbit polyclonal anti-met-enk (Millipore AB5026, 1:1000) antibodies. Secondary antibodies were AlexaFluor 647-conjugated donkey anti-sheep (Molecular Probes A-21448, 1:500) for CGRP, Dylight 650-conjugated goat anti-rat (Thermofisher SA5-10021, 1:500) for SST, AlexaFluor 488-conjugated goat anti rabbit (Molecular Probes A-11034, 1:2000) for metenk. For colocalization studies between mu-Cherry and CGRP or SST, the fluorescent signal associated with the mu-mCherry fusion construct was amplified using rabbit polyclonal antidsred (Clontech 632496, 1:1000) and AlexaFluor 594-conjugated goat anti rabbit (Molecular Probes A-11012, 1:2000) antibodies. The absence of cross-reactivity (rabbit/sheep, rabbit/rat) was checked in control experiments. Immunohistochemistry was also performed without primary antibodies to verify absence of non-specific staining by the secondary antibody alone. 


\section{Data acquisition, analysis and iconography}

Image acquisition was performed with the slide scanner NanoZoomer S60 and fluorescence module L11600-21 (Hamamatsu Photonics, Japan). The light source LX2000 (Hamamatsu Photonics, Japan) consisted in an ultra-high-pressure mercury lamp coupled to an optical fiber. Acquisitions were made in the epifluorescence mode with a filter-set optimized for DAPI, fluorescein, tetramethylrhodamine or Cy5 detection. The acquisitions were performed using a dry 20x objective (NA: 0.75). The 40x resolution was achieved with a lens converter. The latter mode used the full capacity of the camera (resolution: $0.23 \mu \mathrm{m} / \mathrm{pixel}$ ). Neurons expressing a given fluorescent marker were visualized using the NDP viewer system with an integrated high-resolution zoom and possibility to separate the different fluorescent components. The localization and semiquantitative analysis of the density of CTB retrogradely labeled neurons and BDA anterogradely labeled axons and terminals were carried out using NDP viewer. For illustration purpose, micrographs were taken from the original sections with a Microfire camera (Optronics) on an epifluorescence Nikon Eclipse $80 \mathrm{i}$ microscope. Confocal images were acquired (SP5RS, Leica) with the LCS (Leica) software using a 20x (NA: 1.25) dry objective and a 63x (NA 1.4) oil immersion objective. Confocal acquisitions were performed in the sequential mode (single excitation beams: 405 , 488, 568 and $633 \mathrm{~nm}$ ) to avoid potential crosstalk between the different fluorescence emissions.

The nomenclature used is the one from Paxinos and Franklin's mouse brain atlas (2012) (Paxinos and Franklin 2012).

\section{RESULTS}

\section{Mu opioid receptor expression in the mouse LHsf}

Figure 1 shows the distribution of Mu opioid receptor-expressing neurons in the $\mathrm{LH}$ of a MumCherry knock-in mouse. Starting rostrally from a diffuse distribution between $-0.83 \mathrm{~mm}$ and $-0.95 \mathrm{~mm}$ to the Bregma, mu-mCherry positive (mu-mCherry+) neurons became concentrated between the fornix (f) and the optic tract (ot) (Fig. 1a, b). This distinctive cluster of neurons form a cap surrounding the ventromedial aspect of the fornix (Fig. 1b-e) and disappeared progressively about $-1.43 \mathrm{~mm}$ to the Bregma (Fig. 1f). The term "subfornical region of the LH" (LHsf) has been thus used to define this specific region in which we targeted our tracer injection, even though it may slightly differ from the LHsf area defined in rats (see discussion). 


\section{LH injection sites}

Evaluation of the injection sites, using the mouse brain atlas of Paxinos and Franklin (Paxinos and Franklin 2012), allowed selecting four mice with correct CTB injections (Figs. 2a and 3a-d), and five mice with correct BDA injections (Figs. 2b and 3e-i), within the LHsf region. The mCherry signal was used to ascertain the correct localization of the injection sites within the boundaries of the region of the LHsf expressing mu opioid receptors (Fig. 3).

\section{Retrograde labeling of LHsf afferents}

Analysis of brain sections from mice receiving CTB injections in the LHsf revealed CTBpositive $(\mathrm{CTB}+)$ cells mainly in ipsilateral structures from the frontal cortex to the caudal pons. (Figs. 4-8). We did not observe any major differences in the localization and relative density of the retrograde labeling between individual CTB cases.

Only a few cortical areas contribute to LHsf afferents. The anterior cingulate area A24a displayed a moderate density of CTB+ cells, especially in its rostral part anterior to the genu of the corpus callosum (Fig. 4a,b). At similar level, a low number of CTB+ cells were observed in the anterior cingulate area A25 and in the ventral (VO) and medial orbital cortices (MO) (Fig. 4a,b). Additionally, the cingulate area A24b, the piriform (Pir) and insular (AI) cortices displayed a few scattered CTB+ somas. No labeled cells could be detected in the hippocampal region.

In the rostral forebrain, the dorsal taenia tecta (DTT) contained a low number of retrogradely labeled cells (Fig. 4a). More caudally, CTB+ somas with a light to moderate density were systematically observed in the dorsomedial shell of nucleus accumbens (AcbSh; Fig. 4c,d). Dorsally, the LS contained numerous CTB+ neurons, with a very high density in the intermediate part (LSI), especially laterally (Fig. 4e,f). The dorsal (LSD) and ventral (LSV) parts displayed a lower density of labeling, as well as the adjacent septohypothalamic nucleus (SHy); Fig. 4e). Within the medial division of the bed nuclei of the stria terminalis (BST), the anterior and ventral parts (BSTMA, BSTMV) exhibited a light density of CTB+ neurons whereas a moderately dense labeling was observed in the posterior medial BST (BSTMP). Of note, no CTB+ neurons were detected in the lateral BST. Caudally, the amygdala displayed retrogradely labeled neurons in several nuclei. The medial nucleus (MeA) harbored a moderate to strong density of CTB+ cells in its anteroventral (MeAV; Fig. 5a,b), and posterodorsal (MePD; Fig. 5 c-f) parts, with only a light labeling in the anterodorsal (MeAD) and posteroventral (MePV; Fig. 5 a-f) parts. The associated intraamygdaloid division of the 
BST (BSTIA) also contained a light to moderate number of CTB+ cells (Fig. 5c-f). In the pallial amygdala, the highest density of retrograde labeling was observed in the posterior part of the basolateral nucleus (BLP; Fig. 5g,h) whereas the basomedial nucleus displayed a light density of CTB+ cells in its anterior and posterior parts (BMA, BMP; Fig. 5). Finally, the anterior amygdalohippocampal area (AHiA) contained a light density of $\mathrm{CTB}+$ cells and a more sporadic labeling was observed in the anterior amygdaloid area (AAA), the anterior and posteromedial cortical nuclei (ACo, PMCo) and the caudalmost region of the lateral nucleus (La) (Fig. 5).

Inputs from the thalamus appeared very sparse, as only a low density of $\mathrm{CTB}+$ neurons were detected in the posterior intralaminar and posterior triangular nuclei. In addition, occasional $\mathrm{CTB}+$ neurons were detected in the zona incerta $(\mathrm{ZI})$.

In the hypothalamus, $\mathrm{CTB}+$ cells were found in several nuclei/areas, especially in the periventricular and medial zones. In the preoptic region, the medial preoptic nucleus (MPO) and area (MPA) contained a light to moderate density of CTB+ cells, while the density was lighter in the lateral preoptic area (LPO; Fig. 6ab). At anterior level, the labeling was sporadic in the anterior hypothalamic area $(\mathrm{AH})$, but distinctive concentrations of CTB+ neurons were observed in the supraoptic nucleus (SO) and the paraventricular nucleus (Pa), mostly in the lateral magnocellular part (Fig. 6c,d). More caudally, two nuclei displayed a dense concentration of retrogradely labeled cells. At tuberal level, the ventromedial nucleus (VMH) contained numerous $\mathrm{CTB}+$ cells without obvious preferential repartition in its subdivisions (Fig. 7a-c), while rare labeled cells were scattered in the dorsomedial nucleus (DM). In the mamillary region, a high density of CTB+ cells was observed in the dorsal premamillary nucleus (PMD), mostly in its ventral region, whereas the labeling appeared light in the posterior hypothalamus $(\mathrm{PH})$ and only occasional in the ventral premamillary (PMV) and retromamillary (RM) nuclei (Fig. 7d,e). In the LH itself, the halo of the injection sites in LHsf did not allow identifying a possible retrograde labeling in other LH subdivisions.

In the brainstem, $\mathrm{CTB}+$ cells were mainly detected in the midbrain and in the mesopontine tegmentum (Fig. 8). The periaqueductal gray (PAG) contained a light to moderate density of $\mathrm{CTB}+$ somas in the rostral (rPAG) and dorsolateral PAG (dlPAG), while labeling appeared lighter in the lateral PAG (IPAG) and more sporadic in the dorsolateral (dlPAG) and ventrolateral (vlPAG) columns (Fig. 8ab). Among the raphe nuclei, the median/paramedian nuclei (MnR/PMnR) displayed a light retrograde labeling, while the dorsal (DR) and rostral linear nucleI (RLi) contained only a few $\mathrm{CTB}+$ cells. In the mesopontine region, a low 
density of $\mathrm{CTB}+$ neurons could be observed in the cuneiform nucleus $(\mathrm{CnF})$, but the medial paralemniscal nucleus (MPL) harbored a moderate to high density of retrogradely labeled neurons (Fig. 8c-e). Occasional CTB+ neurons were observed in tegmental nuclei such as the laterodorsal (LDTg), and pedunculotegmental (PTg) nuclei. In the parabrachial area, the CTB labeling in the central lateral (LPBC; Fig. 8f) and superior lateral (LPBS) nuclei appeared moderately and lightly dense, respectively. Finally, in the caudodorsal pons, CTB+ neurons were constantly observed with a low density in the central gray, alpha and beta parts (CGA, CGB; Fig. 8g) and some isolated CTB+ neurons were sporadically observed in the region of locus coeruleus (LC) and Barrington's nucleus (Bar).

\section{Anterograde labeling of LHsf efferents}

The analysis of brain sections from mice receiving BDA injections in LHsf revealed BDApositive $(\mathrm{BDA}+)$ fibers and terminals mainly in ipsilateral structures from the frontal region to the caudal pons (Figs. 9-15). As in the case of the CTB labeling, we did not observe any major differences in the localization of the anterograde labeling between individual BDA cases. Injection sites in cases BDA M-B (Fig. 3h) and BDA M-H (Fig. 3i) were more restricted than for the three other cases, and the overall density of anterograde labeling was lower, but not qualitatively different.

Cortical areas, including the hippocampal region, were devoid of anterograde labeling, with the exception of single BDA+ axons sporadically observed in area A24a.

The septal region exhibited the densest labeling, with a very dense plexus of BDA+ fibers and terminals in the LSI (Fig. 9a-c, e), in the same region that exhibited CTB+ somas. The terminals are often organized in pericellular baskets apparently surrounding somas and proximal dendrites of LSI neurons (Fig. 9e). This axonal plexus extends ventrally in the LSV with a reduced density (moderate to dense) and in the SHy with a light density (Fig. 9a-c). Dorsally, the LSD appeared almost devoid of BDA+ fibers. The medial septum (MS) displayed a moderate to high density of vertically oriented BDA+ axons, which continued their course caudoventrally in the vertical limb of the diagonal band of Broca (VDB) (Fig. 9a, $b, g)$. At the same level, a few axons systematically spread out in the dorsomedial tip of the AcbSh, sometimes invading the AcbC (Fig. 9d, f). In the BST, some rare labeled axons were observed in the posterior part of the lateral division (BSTLP), and in the medial division (BSTMA, BSTMV; Fig. 10a,b). More caudally, the BSTMP contained a more constant, but light, density of BDA+ axons in the posterolateral and posterointermediate parts (BSTMPL/I), but not in the posteromedial part (BSTMPM; Fig. 10c). 
In the amygdala (Fig. 11), a sporadic or light innervation was observed in a few nuclei such as the AAA, MePD, BSTIA, La, BLA and AHiA, but the BMP displayed a higher density of BDA+ axons in its caudal aspect (Fig. 11g, h). The most striking feature of the BDA labeling in the amygdala was the almost systematic presence of BDA+ axons in, or close to, the intercalated nuclei (I), especially those located at the interface between the basolateral complex and the central nucleus and the BSTIA (Fig. 11a-e), but also between the basolateral and lateral nuclei (Fig. 11c-e) and at the border of the main intercalated nucleus (Im; Fig. 11f).

While thalamic inputs to the LHsf appeared meager, several thalamic nuclei displayed BDA+ axons stemming from the LHsf, especially in the midline group. The paratenial (PT), paraventricular (PV) and reuniens (Re) nuclei contained a light to moderate density of labeled axons (Fig. 12a-c). Additionally, isolated BDA+ fibers could be seen in the mediodorsal (MD) and centromedial (CM) nuclei. Ventrally, the ZI also harbored a light density of BDA+ axons. In the epithalamus, a dense plexus of BDA+ fibers was observed in the lateral habenula ( $\mathrm{LHb})$, mainly in its medial part (LHbM; Fig. 12d-i). The most lateral part of the lateral $\mathrm{LHb}(\mathrm{LHbL})$, possibly corresponding to the oval subnucleus, was almost devoid of $\mathrm{BDA}+$ fibers in the rostral two-thirds of the nucleus, but the overall labeling was more expansive caudally (Fig. 12h,i). A lighter labeling was also noted in the controlateral LHb.

In the hypothalamus, the recipients of LHsf afferents were observed mostly in the medial zone. Rostrally, the MPA and the AH displayed a light to moderate density of BDA+ fibers (Fig. 10a,d-h). The Pa contained only sporadic labeling. At tuberal level, a moderate to high density of BDA+ axons were present in the VMH (Fig. 13). At caudal level, labeled axons were concentrated mostly in the central part of the nucleus (VMHC; Fig. 13a-c), while more caudally, the labeling also extended in dorsomedial (VMHDM) and ventrolateral parts (VMHVL; Fig. 13d-f). In the mamillary region, a dense BDA+ axonal plexus was observed in the PMD, mostly in its dorsal part (Fig. 13g,j). At the same level, the medial retromamillary nucleus (RMM) and PH contained a light density of BDA+ axons (Fig. 13g,i). As for retrograde tracing experiments, the saturation of LH itself with thin labeled axons did not allow for an unequivocal identification of LHsf efferent to other LH subdivisionsThe overall anterograde labeling observed in the brainstem was modest. In the ventral midbrain, BDA+ axons invaded the region of the ventral tegmental area (VTA) with a light to moderate density in the different subregions (Fig. 14), namely the rostral VTA (VTAR), the parabrachial pigmented nucleus $(\mathrm{PBP})$, the paranigral nucleus $(\mathrm{PN})$ and the parainterfascicular nucleus (PIF). At the same level, the RM displayed a light innervation, 
and some isolated axons were found in the medial nuclei of the mamillary bodies (MM, ML; Fig. 14a,b). In the PAG, BDA+ axons entered the rostral PAG in a with vertical ventrodorsal organization with a moderate density (Fig. 13g,h). More caudally, the axons spread laterally with a light to moderate density in the $\mathrm{PAG}$ and $\mathrm{dmPAG}$, but the density appeared very low in the dIPAG (Fig. 15a,b). In the caudal third of the PAG, a light density of axons persisted in the IPAG and vlPAG (Fig. 15c,d). At this level, the DR contained a light density of BDA+ axons mostly in the ventral and dorsal parts (DRV, DRC; Fig. 15f). Ventrally, the MnR/PMnR region displayed a light innervation (Fig. 15e).Finally, in the caudal pons, single BDA+ axons were observed in the region of CGA, CGB, LC and Bar (Fig. 15g,h).

\section{Neurochemistry}

Immunohistochemical labeling with various neuronal hypothalamic markers did not reveal any co-localization in the LHsf between mu-mCherry neurons and orexin, melaninconcentrating hormone, parvalbumin, calretinin, acetylcholine-esterase, substance $\mathrm{P}$ or tyrosine hydroxylase (data not shown). However, a significant proportion of the mu-mCherry neurons co-localized with CGRP (Fig. 16a-d) and a smaller fraction with SST (Fig. 16e-g).

Anterograde labeling of the LHsf following BDA injection in the LS revealed BDA+ terminals in close proximity to mu-mCherry neurons (Fig. 17a-c). Reciprocally, retrograde labeling of the LS following CTB injection in the LHsf revealed that the LS neurons projecting to the LHsf are in part mu-mCherry $+($ Fig. 17d-f). This indicates that a fraction of the reciprocal LS-LHsf connection involved mu opioid receptor expressing neurons in both structures and suggests a possible opioidergic modulation of these pathways..

We then tried to identify neurons expressing opioid peptides that would contact mu-mCherry neurons and therefore could negatively modulate the activity of this loop through mu opioid receptor activation. We focused on enkephalinergic terminals because of the high level of this peptide in the LH (Sukhov et al. 1995) and the previously suggested enkephalinergic projections of the LHsf onto the LS (Sakanaka and Magari 1989) Also, we did not identify any significant input from the Arc on the LHsf, which does not suggest any major contribution of $\beta$-endorphin. Accordingly, immunoreactive enkephalinergic terminals were observed in close apposition to mu-mCherry neurons in the LS, including those projecting to LHsf (Fig. 17d-h). In the LHsf, some projections from CGA/CGB are also likely enkephalinergic. Indeed, anterograde tracing with BDA injection in the CGA produced terminals in close contact with mu-mCherry neurons (Fig. 18). 


\section{DISCUSSION}

In this work, we identified the connections of a subregion of the mouse subfornical lateral hypothalamus (Fig. 19). Importantly, the region we targeted and identified as the LHsf has been recognized by a distinctive concentration of neurons expressing the mu opioid receptor. Thus, while these neurons are indeed located in a region ventral to the fornix, justifying the term "subfornical", it does not mean that the boundaries of the mouse LH subfornical area are strictly equivalent to those defined by the expression of the mu opioid receptor, nor that the mouse LHsf is topographically equivalent to the rat LHsf. Of note, no detailed distribution of the mu opioid receptor in the LH has been reported so far. Therefore, it is important to compare our results with those obtained in the rat not only for the LHsf but also for adjacent LH subregions. Indeed, our comprehension of the topography of the mouse LH is not as advanced as in the rat, for which a detailed parcellation has been proposed (Swanson 2004). According to this topographical description, the rat LHsf is bordered medially by the juxtaventromedial part of the LH (LHAjv) and laterally by the anterior (LHAa) and dorsal (LHAd) parts of the LH. In addition, a distinction is made between the anterior (LHAsfa) and posterior (LHAsfp) parts of the LHsf, based on a dense input from the nucleus incertus (NI) to the LHAsfa (Goto et al. 2001; Goto et al. 2005). The connections of most of these regions have been comprehensively analyzed by the group of Larry Swanson (Goto et al. 2005; Hahn and Swanson 2010, 2015).We will therefore use the abbreviation LHsf for the region we analyzed in mice, whereas the regions of the rat lateral hypothalamic area (LHAsfa, LHAsfp, LHAa, LHAd and LHAjv) will be named according to Swanson's nomenclature (Swanson 2004).

\section{Comparison between mouse and rat neuroanatomical organization}

Most of the connections reported here for the LHsf are comparable in mice and rats, especially for quantitatively significant connections (LS, MS, VMH, PMD, LHb) with a few interspecific differences.

The mouse LHsf receives a modest cerebral input, mainly from the rostral cingulate areas, with almost no reciprocal pathway. This is consistent with the connectivity described in rats (Goto et al. 2005; Hahn and Swanson 2015; Vertes 2004), despite differences in nomenclature. It is also in agreement with the previously reported efferents of the mouse anterior cingulate areas (Fillinger et al. 2018).

The septal region is a major partner of the LH (Risold and Swanson 1997). The robust reciprocal connections we described in the LHsf, favoring the lateral part of the LSI, are very 
comparable to those reported for the rat LHAsfa, with the characteristic pericellular baskets formed in the LS by the hypothalamic terminals (Risold and Swanson 1997; Goto et al. 2005). Similarly, the dense projection of the LHsf to the MS is also found in its rat counterpart (Goto et al. 2005), but we did not identify any significant input from the MS to the LHsf whereas it seems to exist in rats (Swanson and Cowan 1979).

We observed a reciprocal connection between the LHsf and the AcbSh. The density of retrogradely labeled neurons and anterogradely labeled terminals remained overall modest, but reproducible, and occurred specifically in the dorsomedial AcbSh. This is in agreement with the targeting of the rat LHAa by the dorsomedial tip of AcbSh (Thompson and Swanson 2010; Zahm et al. 2013) and the sporadic innervation of the rat AcbSh by the LHAsfa, LHAjv, LHAd, and LHAsfp (Goto et al. 2005).

In the pallial amygdala, the BLP, BMA and BMP project to the LHsf, which, in turn, targets mostly the BMP. The rat LHsfa also projects specifically to the BMP (Goto et al. 2005) and some inputs from the BMA, BMP and BLP have been noted in the ventral $\mathrm{LH}$, including the LHAjv region (Petrovich et al. 1996; Hahn and Swanson 2015; Reppucci and Petrovich 2016). In the extended amygdala, we observed LHsf connections with the medial, but not central, extended amygdala. The reciprocal connections of the LHsf with the subdivisions of the BSTMP appears similar to those of the rat LHsfa (Dong and Swanson 2004; Goto et al. 2005), but the input from the medial amygdala that we observed in the LHsf, especially from the MeAV, seems to be absent in the rat, as medial amygdala rather projects to the LHAjv in this species (Canteras et al. 1995; Hahn and Swanson 2015). Finally, the specific LHsf projection to the intercalated nuclei (I) has also been observed for the rat LHAsfa (Goto et al. 2005).

The mouse LHsf receives a very limited thalamic input, but projects, at least lightly, to several nuclei of the dorsal thalamus associated with limbic functions such as the PT, PV, Re, $\mathrm{CM}$ and MD, as shown in rats (Goto et al. 2005; Vertes et al. 2015). Similarly, the robust projection to the $\mathrm{LHb}$ in mice is very comparable to the pattern of $\mathrm{LHb}$ afferents from the rat LHAsfa (Goto et al. 2005).

The intrahypothalamic projections account for some of the densest connections of the LHsf. Robust reciprocal connections were found with the VMH and the PMD, along with lighter afferents from the Pa, SO, MPA and efferents to the AH and LPO. The outputs to the VMH appeared denser in the central part than in the dorsomedial and ventrolateral parts, by contrast with the rat LHAsfa which favors the dorsomedial part (Goto et al. 2005), while the LHAjv shares a bidirectional connectivity with the entire VMH (Hahn and Swanson 2015). We also 
observed a topographic pattern of connectivity between the mouse LHsf and the PMD, congruent with the connections reported in rats (Goto et al. 2005; Comoli et al. 2000). Afferents to the LHsf originate in the ventral part of the PMD, but the LHsf preferentially projects to the dorsal part of the PMD. Interestingly, Goto et al. (Goto et al. 2005) showed that the vertical limb of the LHAsfa, located just below the fornix, projects only to the dorsal PMD, whereas the horizontal limb, located more ventrally and extending laterally, projects to the ventral PMD. This suggests that the mu opioid receptor positive area of the mouse LHsf would be more related to the vertical limb of the rat LHAsfa.

We did not attempt to disclose the intrinsic connectivity of LHsf with the rest of the LH. Firstly, the parcellation of the mouse LH is not as accurately described as in the rat and it would be premature to define homologous subregions in the absence of comprehensive cytoarchitectural and neurochemical studies. Secondly, our injection sites are indeed restricted to the region expressing the mu opioid receptor, but the surrounding halo of tracers tended to obscure a potential labeling in surrounding regions. While this situation precludes any conclusion concerning intra-LH connectivity, it might also moderate the interpretation of the observed connections with the VMH and SO. Indeed, the closeness of the VMH to the halo of CTB injection sites, as well as the fact that the dendrites of VMH neurons exceed the lateral boundaries of the nucleus (Millhouse 1973; Hahn and Swanson 2015), could lead, at least partially, to non-specific labeling. However, this hypothesis would be less pertinent for the labeling observed in the central and dorsomedial parts of the VMH.

In the brainstem, we found that the PAG inputs arise mainly from the rostral part and the dorsolateral column, similarly to the situation in the rat (Goto et al. 2005)(Kincheski et al. 2012). In a complementary pattern, LHsf efferents favor the rostral PAG and the lateral and ventrolateral columns but tend to avoid the dorsolateral column. Interestingly, a similar poor innervation of the dIPAG has been observed in the rat from LHAsfa, but also from LHAsfp, LHAjv and LHAd (Goto et al. 2005; Hahn and Swanson 2015). Besides the PAG, the connections with the MnR, DR and parabrachial nuclei are consistent in the two species (Goto et al. 2005; Vertes et al. 1999; Bester et al. 1997). The moderate LHsf output to the VTA was not reported for the rat LHAsfa but was described for the LHAd and LHAsfp (Goto et al. 2005). On the other hand, we did not identify any significant input from the interpeduncular nucleus, as reported in rats (Groenewegen et al. 1986).

The strong afferent from the MPL to the LHsf has never been reported although the adjacent ventral nucleus of the lateral lemniscus projects to the LHAjv in rats (Hahn and Swanson 2015). The efferents of the MPL have never been specifically explored, but the MPL, along 
with the posterior intralaminar thalamic complex, appears to be the main source of tuberoinfundibular peptide of 39 residues (TIP39), which has been detected in the rat subfornical LH (Dobolyi et al. 2010). The afferent from central gray region in the caudal pons (CGA, CGB) to the mouse LHsf appears rather meager when compared to the dense input to the LHAsfa provided by the NI, the rat homologue of the CGA/CGB (Goto et al. 2005; Goto et al. 2001). Yet, we identified terminals from CGA/CGB axons in close proximity to the LHsf neurons expressing the mu opioid receptor, pointing to a direct control by the CGA/CGB neurons which can be enkephalinergic. Further studies are needed to explore in more detail a potential functional difference between the two species.

\section{Neurochemical features of the mouse LHsf}

In addition to the definition of the connectivity, our preliminary neurochemical study provides additional insight on the LHsf organization. We observed that a significant part of the neurons expressing the mu opioid receptor in the LHsf were immunoreactive for CGRP and a lesser proportion for SST. Dobolyi et al. (Dobolyi et al. 2005) reported, in rats, the presence of neurons immunoreactive for CGRP in the perifornical hypothalamus, as well as CGRP+ fibers in the LSI, with a pattern resembling the LHAsfa projections. Interestingly, they found that lesioning this LH region led to the complete disappearance of the CGRP innervation in the LSI, but not in the rest of the brain. These data suggest that, at least in rats, the LSI is the main target of LHAsfa neurons expressing CGRP. If the situation is similar in mice, it would mean that the LHsf CGRP/Mu-mCherry neurons are essentially involved in projections to the LSI, while other LHsf efferents would arise from neurons with a different neurochemical phenotype. For example, the PMD is a major target of the LHsf, but does not seem to contain CGRP+ fibers (Lantos et al. 1995). On the other hand, the enkephalinergic neurons in the PMD (Lantos et al. 1995) could contribute to the modulation of neurons expressing the mu opioid receptor in the LHsf. Additionally, an enkephalinergic modulation of the LHsf can be exerted by the CGA/CGB, despite its modest input, but opioidergic control could also arise from other afferents expressing enkephalin such as the VMH (Williams and Dockray 1983)(Sakanaka et al. 1989). Finally, an indirect opioidergic control of LHsf could be achieved through LS/LHsf projections, since we found that the LHsfprojecting neurons could also express mu opioid receptors and are potentially contacted by enkephalinergic terminals. Thus, the presence of mu opoid receptors and/or opioidergic neurons in the LHsf network suggests a strong opioidergic control in LHsf functions. 


\section{Functional considerations}

From a functional point of view, the $\mathrm{LH}$ as a whole is thought to act as a central coordinator of behavioral control, with the mosaic of identified regions being more specialized. For example, LHAs appears to be largely involved in ingestive behaviors, juxtaperiventricular and juxtadorsomedial regions in defensive behaviors, while LHAjv would control reproduction-related behaviors, in addition to a more general "high-level" coordinating role (Hahn and Swanson 2015). In this context, the LHAsfa has been suggested to "help matching adaptive behavioral responses (either defensive or foraging) to current internal motivational status and external environmental conditions" (Goto et al. 2005). This conclusion was based on the analysis of the LHAsfa connections in the rat and we refer the reader to the detailed discussion in (Goto et al. 2005). Briefly, the LHAsfa is strongly connected to (1) a midline brainstem network, consisting in NI, MnR and interpeduncular nucleus (IPN), modulating behavioral arousal (Goto et al. 2001), (2) a medial hypothalamic circuit, consisting in $\mathrm{AH}$, VMH and PMD, controlling defensive behaviors (Canteras 2002), and (3) a set of major forebrain structures (LSI, MS, BSTM, BMP, I, LHb) that contribute to the control and/or modulation of defensive and foraging reactions through (poly)sensory, motivational and emotional information. In mice, despite the absence of connections with the IPN and the weaker input with CGA/CGB, the core of the LHsf circuitry is largely in accordance with a role in defensive and foraging behaviors. Indeed, the exposure of mice to a predator (rat) induces an activation of the medial hypothalamic defense system, but also of the LHsf (Martinez et al. 2008). Moreover, some of the specific inputs found in mice but not in rats can also be consistent with these functions, such as inputs from the MeAV, involved in agonistic/defensive functions (Canteras et al. 1995) or from the MPL which could participate in defensive reactions to alerting sounds (Dobolyi et al. 2010).

Part of the connections common to mice and rats, as well as some specific connections of the mouse LHsf, namely with the AcbSh, also suggest a potential implication in appetitive and consummatory behaviors, in line with the involvement of the opioid system in these behaviors (Bodnar 2019). The LHsf is reciprocally connected to the AcbSh, in particular with the dorsomedial tip corresponding a very discrete area, identified in rats as a hedonic hotspot (Pecina and Berridge 2005). This region has been shown to project massively to the rat LHAa (Thompson and Swanson 2010). In addition, a reciprocal connection of this region of the AcbSh with the LHAjv also exists (Hahn and Swanson 2015). Considering the potential interspecific differences in the anatomo-functional organization of the $\mathrm{LH}$, it is possible that the mouse LHsf includes, at least in part, some functions supported in rats by other regions 
such as the LHAa or LHAjv. Indeed, Thompson and Swanson (2010) (Thompson and Swanson 2010) showed that the AcbSh-recipient LHAa projects mainly to the PMD, PAG, LHb, PT and VTA, which are also targeted by the mouse LHsf. Two potential indirect reciprocal connections between the AcbSh and the LHsf were also described in rats, through the LS (Zahm et al. 2013) or the ventral pallidum (Urstadt and Stanley 2015). In mice, we observed a massive interconnection between the LHsf and the LS, but we did not identify any significant connections between the LHsf and the VP. Nonetheless, the presence of a direct/indirect AcbSh-LHsf loop strongly suggests a role in food consumption. The roles of LH in feeding and reward have been largely focused on the orexin and melanin-concentrating hormone systems. These neuropeptides are expressed in the LH mostly dorsally, medially and laterally to the fornix, but almost not ventrally (Swanson et al. 2005; Bonnavion et al. 2016). However, it is now clear that non-orexin, non MCH neurons in the LH may also be involved in appetitive and consummatory behaviors, especially GABAergic neurons (Jennings et al. 2015). Recently, food access was reported to increase c-fos levels in the posterior part of the LHsf in rats (Reppucci and Petrovich 2018). This observation is also congruent with the fact that stimulation of the mu opioid receptor by either exogenous opioid agonists or endogenous opioid peptides stimulates food intake (Bodnar 2019). In the LHsf, activation of $\mathrm{mu}$ opioid receptors located on the anorexinergic CGRP- and SST-positive neurons would decrease their activity and therefore could promote food consumption. The $\mathrm{mu}$ opioid receptor/CGRP neurons projecting to the LS will then affect its activity and will modulate its output to the AcbSh. In turn, the activity of the LHsf can be regulated by the LS. Of note, retrograde labeling upon CTB injection in the LHsf shows that the LS inputs include neurons expressing mu opioid receptors under enkephalinergic control. This indicates a dual opioid control of the LHsf activity both locally and through afferents and suggests that endogenous enkephalins could primarily set a background motivational tone regulating feeding behavior (Mendez et al. 2015). Finally, the LHsf output to the VTA could represent an additional control of the motivational aspects of feeding through the mesolimbic pathway. In conclusion, our analysis suggests that the mouse LHsf region characterized by neurons expressing mu opioid receptors corresponds, at least partially, to the rat LHAsfa. This conclusion is supported by the similarity of the main connections in both species. However, a few connections appear more typical of other LH regions and can be interpreted as true interspecies differences or as an indication that the region we described in mice contains elements actually belonging to the rat LHAa, LHAjv or LHAsfp. Accordingly, the mouse LHsf would be involved in the control of defensive and foraging behaviors, as does the rat 
LHAsfa, but also in appetitive and consummatory behaviors. These results underline the necessity of better understanding the organization of mouse LH, as this species has been widely used in functional studies (Berthoud and Munzberg 2011).. 


\section{Acknowledgments}

We thank the Chronobiotron (UMS3415) for animal housing and animal care, and the imaging platform of INCI (UPS3156) for their assistance.

\section{Declarations}

Funding: This work was supported by the Centre National de la Recherche Scientifique (contract UPR3212), the University of Strasbourg and the NeuroTime Erasmus Mundus Joint Doctorate Program.

Conflicts of interest/Competing interests: The authors declare that they have no conflict of interest, nor any competing interest

Availability of data and material: The datasets generated and analysed during the current study are available from the corresponding author on reasonable request.

Code availability: not applicable

Ethics approval: All experiments were approved by the Comité d'éthique en Matière d'expérimentation animale (authorization number 2015304113547b (APAFIS \#300.02)) and conducted in agreement with the European Communities Council Directive 2010/63/EU for animal experiments.

Consent to participate: No human subject were used in this study

Consent for publication: No human subject were used in this study 


\section{References}

Ardianto C, Yonemochi N, Yamamoto S, Yang L, Takenoya F, Shioda S, Nagase H, Ikeda H, Kamei J (2016) Opioid systems in the lateral hypothalamus regulate feeding behavior through orexin and GABA neurons. Neuroscience 320:183-193. doi:10.1016/j.neuroscience.2016.02.002

Baldo BA, Gual-Bonilla L, Sijapati K, Daniel RA, Landry CF, Kelley AE (2004) Activation of a subpopulation of orexin/hypocretin-containing hypothalamic neurons by GABAA receptor-mediated inhibition of the nucleus accumbens shell, but not by exposure to a novel environment. Eur J Neurosci 19 (2):376-386

Berthoud HR, Munzberg H (2011) The lateral hypothalamus as integrator of metabolic and environmental needs: from electrical self-stimulation to opto-genetics. Physiol Behav 104 (1):29-39. doi:10.1016/j.physbeh.2011.04.051

Bester H, Besson JM, Bernard JF (1997) Organization of efferent projections from the parabrachial area to the hypothalamus: a Phaseolus vulgaris-leucoagglutinin study in the rat. J Comp Neurol 383 (3):245-281

Bodnar RJ (2019) Endogenous opioid modulation of food intake and body weight: Implications for opioid influences upon motivation and addiction. Peptides 116:42-62. doi:10.1016/j.peptides.2019.04.008

Bonnavion P, Mickelsen LE, Fujita A, de Lecea L, Jackson AC (2016) Hubs and spokes of the lateral hypothalamus: cell types, circuits and behaviour. J Physiol 594 (22):64436462. doi:10.1113/JP271946

Canteras NS (2002) The medial hypothalamic defensive system: hodological organization and functional implications. Pharmacol Biochem Behav 71 (3):481-491. doi:10.1016/s0091-3057(01)00685-2

Canteras NS, Simerly RB, Swanson LW (1995) Organization of projections from the medial nucleus of the amygdala: a PHAL study in the rat. J Comp Neurol 360 (2):213-245. doi:10.1002/cne.903600203

Comoli E, Ribeiro-Barbosa ER, Canteras NS (2000) Afferent connections of the dorsal premammillary nucleus. J Comp Neurol 423 (1):83-98. doi:10.1002/10969861(20000717)423:1<83::aid-cne7>3.0.co;2-3

Dobolyi A, Irwin S, Makara G, Usdin TB, Palkovits M (2005) Calcitonin gene-related peptide-containing pathways in the rat forebrain. J Comp Neurol 489 (1):92-119. doi:10.1002/cne.20618

Dobolyi A, Palkovits M, Usdin TB (2010) The TIP39-PTH2 receptor system: unique peptidergic cell groups in the brainstem and their interactions with central regulatory mechanisms. Prog Neurobiol 90 (1):29-59. doi:10.1016/j.pneurobio.2009.10.017

Dong HW, Swanson LW (2004) Projections from bed nuclei of the stria terminalis, posterior division: implications for cerebral hemisphere regulation of defensive and reproductive behaviors. J Comp Neurol 471 (4):396-433. doi:10.1002/cne.20002

Erbs E, Faget L, Scherrer G, Matifas A, Filliol D, Vonesch JL, Koch M, Kessler P, Hentsch D, Birling MC, Koutsourakis M, Vasseur L, Veinante P, Kieffer BL, Massotte D (2015) A mu-delta opioid receptor brain atlas reveals neuronal co-occurrence in subcortical networks. Brain Struct Funct 220 (2):677-702. doi:10.1007/s00429-0140717-9

Fillinger C, Yalcin I, Barrot M, Veinante P (2018) Efferents of anterior cingulate areas 24a and $24 \mathrm{~b}$ and midcingulate areas $24 \mathrm{a}^{\prime}$ and $24 \mathrm{~b}^{\prime}$ in the mouse. Brain Struct Funct 223 (4):1747-1778. doi:10.1007/s00429-017-1585-x

Georgescu D, Zachariou V, Barrot M, Mieda M, Willie JT, Eisch AJ, Yanagisawa M, Nestler EJ, DiLeone RJ (2003) Involvement of the lateral hypothalamic peptide orexin in morphine dependence and withdrawal. J Neurosci 23 (8):3106-3111 
Goto M, Canteras NS, Burns G, Swanson LW (2005) Projections from the subfornical region of the lateral hypothalamic area. J Comp Neurol 493 (3):412-438. doi:10.1002/cne.20764

Goto M, Swanson LW, Canteras NS (2001) Connections of the nucleus incertus. J Comp Neurol 438 (1):86-122

Groenewegen HJ, Ahlenius S, Haber SN, Kowall NW, Nauta WJ (1986) Cytoarchitecture, fiber connections, and some histochemical aspects of the interpeduncular nucleus in the rat. J Comp Neurol 249 (1):65-102. doi:10.1002/cne.902490107

Hahn JD, Swanson LW (2010) Distinct patterns of neuronal inputs and outputs of the juxtaparaventricular and suprafornical regions of the lateral hypothalamic area in the male rat. Brain Res Rev 64 (1):14-103. doi:10.1016/j.brainresrev.2010.02.002

Hahn JD, Swanson LW (2015) Connections of the juxtaventromedial region of the lateral hypothalamic area in the male rat. Front Syst Neurosci 9:66. doi:10.3389/fnsys.2015.00066

Jennings JH, Ung RL, Resendez SL, Stamatakis AM, Taylor JG, Huang J, Veleta K, Kantak PA, Aita M, Shilling-Scrivo K, Ramakrishnan C, Deisseroth K, Otte S, Stuber GD (2015) Visualizing hypothalamic network dynamics for appetitive and consummatory behaviors. Cell 160 (3):516-527. doi:10.1016/j.cell.2014.12.026

Kincheski GC, Mota-Ortiz SR, Pavesi E, Canteras NS, Carobrez AP (2012) The dorsolateral periaqueductal gray and its role in mediating fear learning to life threatening events. PLoS One 7 (11):e50361. doi:10.1371/journal.pone.0050361

Lantos TA, Gorcs TJ, Palkovits M (1995) Immunohistochemical mapping of neuropeptides in the premamillary region of the hypothalamus in rats. Brain Res Brain Res Rev 20 (2):209-249. doi:10.1016/0165-0173(94)00013-f

Martinez RC, Carvalho-Netto EF, Amaral VC, Nunes-de-Souza RL, Canteras NS (2008) Investigation of the hypothalamic defensive system in the mouse. Behav Brain Res 192 (2):185-190. doi:10.1016/j.bbr.2008.03.042

Mendez IA, Ostlund SB, Maidment NT, Murphy NP (2015) Involvement of Endogenous Enkephalins and beta-Endorphin in Feeding and Diet-Induced Obesity. Neuropsychopharmacology 40 (9):2103-2112. doi:10.1038/npp.2015.67

Millhouse OE (1973) The organization of the ventromedial hypothalamic nucleus. Brain Res 55 (1):71-87

Paxinos G, Franklin KBJ (2012) Paxinos and Franklin's the mouse brain in stereotaxic coordinates 4th edition. Academic Press,

Pecina S, Berridge KC (2005) Hedonic hot spot in nucleus accumbens shell: where do muopioids cause increased hedonic impact of sweetness? J Neurosci 25 (50):1177711786. doi:10.1523/JNEUROSCI.2329-05.2005

Petrovich GD, Risold PY, Swanson LW (1996) Organization of projections from the basomedial nucleus of the amygdala: a PHAL study in the rat. J Comp Neurol 374 (3):387-420. doi:10.1002/(SICI)1096-9861(19961021)374:3<387::AIDCNE6>3.0.CO;2-Y

Reppucci CJ, Petrovich GD (2016) Organization of connections between the amygdala, medial prefrontal cortex, and lateral hypothalamus: a single and double retrograde tracing study in rats. Brain Struct Funct 221 (6):2937-2962. doi:10.1007/s00429-0151081-0

Reppucci CJ, Petrovich GD (2018) Neural substrates of fear-induced hypophagia in male and female rats. Brain Struct Funct 223 (6):2925-2947. doi:10.1007/s00429-018-1668-3

Risold PY, Swanson LW (1997) Connections of the rat lateral septal complex. Brain Res Brain Res Rev 24 (2-3):115-195 
Sakanaka M, Magari S (1989) Reassessment of enkephalin (ENK)-containing afferents to the rat lateral septum with reference to the fine structures of septal ENK fibers. Brain Res 479 (2):205-216

Sakanaka M, Magari S, Shibasaki T, Inoue N (1989) Co-localization of corticotropinreleasing factor- and enkephalin-like immunoreactivities in nerve cells of the rat hypothalamus and adjacent areas. Brain Res 487 (2):357-362

Sukhov RR, Walker LC, Rance NE, Price DL, Young WS, 3rd (1995) Opioid precursor gene expression in the human hypothalamus. J Comp Neurol 353 (4):604-622. doi:10.1002/cne.903530410

Swanson LW (2004) Brain Maps: Structure of the Rat Brain. A Laboratory Guide with Printed and Electronic Templates for Data, Models and Schematics. 3rd edn. Elsevier, Amsterdam

Swanson LW, Cowan WM (1979) The connections of the septal region in the rat. J Comp Neurol 186 (4):621-655. doi:10.1002/cne.901860408

Swanson LW, Sanchez-Watts G, Watts AG (2005) Comparison of melanin-concentrating hormone and hypocretin/orexin mRNA expression patterns in a new parceling scheme of the lateral hypothalamic zone. Neurosci Lett 387 (2):80-84. doi:10.1016/j.neulet.2005.06.066

Thompson RH, Swanson LW (2010) Hypothesis-driven structural connectivity analysis supports network over hierarchical model of brain architecture. Proc Natl Acad Sci U S A 107 (34):15235-15239. doi:10.1073/pnas.1009112107

Urstadt KR, Stanley BG (2015) Direct hypothalamic and indirect trans-pallidal, transthalamic, or trans-septal control of accumbens signaling and their roles in food intake. Front Syst Neurosci 9:8. doi:10.3389/fnsys.2015.00008

Vertes RP (2004) Differential projections of the infralimbic and prelimbic cortex in the rat. Synapse 51 (1):32-58. doi:10.1002/syn.10279

Vertes RP, Fortin WJ, Crane AM (1999) Projections of the median raphe nucleus in the rat. J Comp Neurol 407 (4):555-582

Vertes RP, Linley SB, Hoover WB (2015) Limbic circuitry of the midline thalamus. Neurosci Biobehav Rev 54:89-107. doi:10.1016/j.neubiorev.2015.01.014

Williams RG, Dockray GJ (1983) Distribution of enkephalin-related peptides in rat brain: immunohistochemical studies using antisera to met-enkephalin and met-enkephalin Arg6Phe7. Neuroscience 9 (3):563-586. doi:10.1016/0306-4522(83)90175-6

Zahm DS, Parsley KP, Schwartz ZM, Cheng AY (2013) On lateral septum-like characteristics of outputs from the accumbal hedonic "hotspot" of Pecina and Berridge with commentary on the transitional nature of basal forebrain "boundaries". J Comp Neurol 521 (1):50-68. doi:10.1002/cne.23157 


\section{LEGENDS}

Fig.1 $\mathrm{Mu}$ opioid receptor expressing neurons in the lateral hypothalamus (LH). a-f: Micrographs, arranged from rostral (a) to caudal (f) showing the distribution of cells expressing Mu-mCherry. Note the conspicuous concentration of Mu-mCherry positive cells in the subfornical region of LH (LHsf). Corresponding Bregma levels from Paxinos and Franklin (2012) are indicated on each panel. See list for abbreviations. Scale bar $=500 \mu \mathrm{m}$.

Fig. 2 Tracer injection sites in the mu opioid receptors-enriched region of the subfornical lateral hypothalamus (LHsf). Micrographs showing two typical injection sites of BDA (a) and of CTB (B) in the LHsf of two Mu-mCherry KI mice. See list for abbreviations. Scale bar= $200 \mu \mathrm{m}$.

Fig. 3 Mapping of $\mathrm{CTB}$ and BDA injection sites. Drawings showing the extent of the injection sites for the four CTB (a-d) and the five BDA (e-i) cases, at three rostro-caudal levels (1 to 3 ). The CTB and BDA deposits are represented by blue and green areas, respectively, with respect to the position of the region of the LHsf enriched in mu opioid receptor positive cells (pink areas).

Fig. 4 Retrograde labeling in the rostral forebrain after CTB injection in the left LHsf. CTB+ cells appear in green. Red boxed areas in a, c, e correspond to b, d, f, respectively. a,b: Distribution of CTB+ neurons in the cingulate areas A24a and A25, in medial (MO) and ventral (VO) orbital cortices and in dorsal tenia tecta (DTT). c,d: Distribution of CTB+ neurons in the nucleus accumbens and the intermediate lateral septal nucleus (LSI). The MumCherry signal (red) and DAPI staining (blue) allow the clear identification of the borders of the shell of the nucleus accumbens (AcbSh) which contains a moderate density of CTB+ cells in its dorsomedial region. e,f: Distribution of CTB+ neurons in the septal region. $(e, f)$ with a denser concentration of $\mathrm{CTB}+$ cells in the lateral LSI, than in the dorsal (LSD) and ventral (LSV) parts of the lateral septum. See list for abbreviation. Scale bars: $a, c, e=500 \mu \mathrm{m} ; \mathrm{b}=$ $200 \mu \mathrm{m} ; \mathrm{d}, \mathrm{f}=50 \mu \mathrm{m}$.

Fig. 5 Retrograde labeling in the amygdala after CTB injection in the left LHsf. CTB+ cells appear in green. Red boxed areas in a, c, e, g correspond to b, d, f, h, respectively. a-f: General labeling in the amygdala at three levels from rostral $(a, b)$ to caudal $(e, f)$. CTB+ cells 
are mostly concentrated in the medial nucleus, especially its anteroventral (MeAV; a, b) and posterodorsal parts (MePD; c-f), with lighter density in the intraamygdaloid division of the BST (BSTIA;c-f), anterior (BMA; a-d) and posterior basomedial nucleus(BMP; e,f). g,h: Labeling in the caudal amygdala. Note the concentration of CTB+ cells in the posterior basolateral nucleus (BLP). See list for abbreviations. Scale bars: a,c,e,g= $500 \mu \mathrm{m} ; \mathrm{b}, \mathrm{d}, \mathrm{f}, \mathrm{h}=$ $100 \mu \mathrm{m}$.

Fig. 6 Retrograde labeling in the rostral hypothalamus after CTB injection in the left LHsf. $\mathrm{CTB}+$ cells appear in green. a: In the preoptic region, the CTB labeling is moderate in the medial preoptic area (MPA) and light in the medial preoptic nucleus (MPO). b corresponds to the red boxed area in a. c-e: In the anterior region, the CTB+ cells are concentrated in the supraoptic ( $\mathrm{SO}$ ) and paraventricular $(\mathrm{Pa})$ nuclei. $\mathrm{d}$ and e correspond to the red boxed areas in c. Note the halo of CTB in the LH, rostrally to the injection site. See list for abbreviations. Scale bars: $\mathrm{a}, \mathrm{c}=500 \mu \mathrm{m} ; \mathrm{b}, \mathrm{d}, \mathrm{e}=50 \mu \mathrm{m}$.

Fig. 7 Retrograde labeling in the caudal hypothalamus after CTB injection in the left LHsf. $\mathrm{CTB}+$ cells appear in green. Red boxed areas in a, b, d correspond to b, c, e, respectively. a-c: In the tuberal region, CTB labeling is concentrated in the ventromedial nucleus (VMH) without preferential distribution in ventrolateral (VMHVL), central (VMHC) or dorsomedial (VMHDM) parts. d,e: In the mamillary region, the labeling is dense in the ventral part of the premamillary nucleus (PMD). See list for abbreviations. Scale bars: $a, d=500 \mu \mathrm{m} ; b=$ $100 \mu \mathrm{m} ; \mathrm{c}, \mathrm{e}=50 \mu \mathrm{m}$.

Fig. 8 Retrograde labeling in the brainstem after CTB injection in the left LHsf. CTB+ cells appear in green. Red boxed areas in a, c, d correspond to b, d, e, respectively. a,b: In the PAG, CTB+ cells are mainly observed in dorsolateral (dlPAG) and lateral (lPAG) columns. c-e: In the caudal midbrain, a moderate/dense CTB labeling is observed in the medial paralemniscal nucleus (MPL). f: Merged image of CTB labeling (green), Mu-mCherry signal (red) and DAPI staining (blue). In the lateral parabrachial nucleus, CTB labeling is moderately dense in the central part (LPBC) and sporadic in other parts, but not in the external part (LPBE) evidenced by expression of Mu-mCherry. g: In the caudal pons, a light CTB labeling is observed in the central gray, part alpha (CGA). See list for abbreviations. Scale bars: $\mathrm{a}, \mathrm{c}=500 \mu \mathrm{m} ; \mathrm{d}, \mathrm{f}=200 \mu \mathrm{m} ; \mathrm{b}, \mathrm{g}=100 \mu \mathrm{m} ; \mathrm{e}=50 \mu \mathrm{m}$. 
Fig. 9 Anterograde labeling in the rostral forebrain after BDA injection in the left LHsf. BDA+ fibers and terminals cells appear in green. a,b: General views of the anterograde labeling at the level of nucleus accumbens and LS at two rostro-caudal levels. The red boxed areas in a correspond to panels $\mathrm{c}, \mathrm{d}$ and g. $\mathrm{c}$ : The intermediate part of the LS (LSI) contained a very high density of BDA+ terminals, extending with a lower density in the ventral part of the LC (LSV; see magnification in e). d: A light density of BDA+ terminals is present in the dorsomedial tip of the shell of nucleus accumbens (AcbSh, detail of the red boxed area in $\mathrm{f}$ ) with some invasion in the core (AcbC). $\mathrm{g}$ : Magnification of the red boxes area in a showing a moderate to high density of BDA+ terminals in the medial septal nucleus (MS). See list for abbreviations. Scale bars: $\mathrm{a}, \mathrm{b}=500 \mu \mathrm{m} ; \mathrm{c}, \mathrm{d}=200 \mu \mathrm{m} ; \mathrm{e}, \mathrm{f}, \mathrm{g}=50 \mu \mathrm{m}$.

Fig. 10 Anterograde labeling in the BST and rostral hypothalamus after BDA injection in the left LHsf. BDA+ fibers and terminals cells appear in green. a: General view of the anterograde labeling at the level of the BST and rostral hypothalamus. b: Magnification of the red boxed area in a, showing scattered BDA+ axons in the BSTLP and BSTMA. c: A more caudal view showing a light labeling in the BSTMPL/I, but not in BSTMPM. d,e: Magnification of the red boxed area in a, showing a light labeling in the MPA and a slightly denser one in the LPO. f: General view of the anterograde labeling at the level of anterior hypothalamus. $g$, h: Magnification of the red boxed area in $\mathrm{f}$ showing a light density of thin labeled axons in the AH. ). See list for abbreviations. Scale bars: a,f= $500 \mu \mathrm{m}$; b,c,d,g= $100 \mu \mathrm{m} ; \mathrm{e}, \mathrm{h}=50 \mu \mathrm{m}$.

Fig. 11 Anterograde labeling in the amygdala after BDA injection in the left LHsf. BDA+ fibers and terminals cells appear in green. a: General view of the anterograde labeling at the level of the mid-caudal amygdala. b: DAPI staining (blue) from the same section than a. Asterisks indicate the position of intercalated nuclei (I). c,d: Magnifications of the red boxed area in $\mathrm{a}$ and $\mathrm{b}$ respectively showing the preferential distribution of BDA+ axons at close proximity of I, identified by dense cellular clusters (asterisks and DAPI staining in c). e: A similar innervation pattern is seen in another section with the visualization of the enriched Mu-mCherry signal in I. f: In a different case, BDA+ axons are seen close to the Im. g,h: At more caudal level, a moderate density of BDA+ axons is observed in the BMP. $\mathrm{h}$ is a magnification of the red boxed area in $\mathrm{g}$. See list for abbreviations. Scale bars: $a, b, g=$ $500 \mu \mathrm{m} ; \mathrm{c}, \mathrm{e}, \mathrm{f}, \mathrm{h}=100 \mu \mathrm{m} ; \mathrm{d}=50 \mu \mathrm{m}$. 
Fig. 12 Anterograde labeling in the thalamus and the habenula after BDA injection in the left LHsf. BDA+ fibers and terminals cells appear in green. a,b: At anterior level, a moderate BDA labeling is observed in the paratenial nucleus (PT) along with a more scattered labeling in the anterior paraventricular nucleus (PVA). c: At more caudal level, the reuniens nucleus (Re) contains a moderate density of BDA+ axons. d-i: Three levels of the habenula are arranged from rostral to caudal. e, f, $g$ correspond to the red boxed areas in $\mathrm{d}, \mathrm{f}, \mathrm{g}$, respectively. A very heavy labeling is apparent in the lateral habenula (LHb), mainly in the medial part $(\mathrm{LHbM})$ in $\mathrm{d}$ and $\mathrm{f}$, but the labeling in the lateral part (LHbL) becomes more extensive at caudal level (h). Note also some axons extending ventromedially in the PV. See list for abbreviations. Scale bars: $\mathrm{a}=500 \mu \mathrm{m} ; \mathrm{b}, \mathrm{c}, \mathrm{d}, \mathrm{f}, \mathrm{h}=200 \mu \mathrm{m} ; \mathrm{e}, \mathrm{g}, \mathrm{i}=50 \mu \mathrm{m}$.

Fig. 13 Anterograde labeling in the caudal hypothalamus after BDA injection in the left LHsf. BDA+ fibers and terminals cells appear in green. a-f: BDA+ axons are observed in the VMH at rostral (a-c) and caudal (d-f) levels, with a preferential distribution in central part (VMHC). Red boxed areas in a, b, d, e correspond to b, c, e,f, respectively. g-j: BDA labeling at premamillary level. $\mathrm{h}, \mathrm{i}, \mathrm{j}$ are magnifications of the corresponding red boxed areas in $\mathrm{g}$ showing the BDA+ labeling with moderate density in the rostral PAG (h), light density in PH (i) and heavy density in the dorsal part of the PMD (j). See list for abbreviations. Scale bars: a,d,g= $500 \mu \mathrm{m} ; \mathrm{b}, \mathrm{e}, \mathrm{h}, \mathrm{i}, \mathrm{j}=100 \mu \mathrm{m} ; \mathrm{c}, \mathrm{f}=50 \mu \mathrm{m}$.

Fig. 14 Anterograde labeling in the ventral tegmental area (VTA) after BDA injection in the left LHsf. BDA+ fibers and terminals cells appear in green. Red boxed areas in a, c correspond to $\mathrm{b}$, d, respectively. a,b: At the caudal pole of the mamillary bodies, BDA+ axonal labeling in the rostral VTA (VTAR), parabrachial pigmented nucleus (PBP) and paranigral nucleus (PN). c,d: At interpeduncular level, BDA+ labeling is lighter in the PBP, but stronger in the PN and the parainterfascicular nucleus (PIF). See list for abbreviations. Scale bars: $a, c=500 \mu \mathrm{m} ; \mathrm{b}, \mathrm{d}=200 \mu \mathrm{m}$.

Fig. 15 Anterograde labeling in the brainstem after BDA injection in the left LHsf. BDA+ fibers and terminals cells appear in green. Red boxed areas in a, c correspond to b, d, respectively. a-d: BDA+ axons in the anterior PAG $(\mathrm{a}, \mathrm{b})$ mostly in lateral (IPAG) and dorsomedial (dmPAG) columns, and in the caudal PAG (c,d), mostly in the ventrolateral column (vlPAG). e: Light BDA labeling in the median (MnR) and paramedian (PMnR) raphe nuclei. f: Moderate labeling in the ventral (DRV) and dorsal (DRD) parts of the dorsal raphe 
nucleus. g,h: In the caudo-dorsal pons, scattered BDA+ axons in the central gray, part alpha and beta (CGA, CGB) and in the region of the locus coeruleus (LC) and Barrington's nucleus (Bar). See list for abbreviations. Scale bars: $\mathrm{a}-\mathrm{h}=200 \mu \mathrm{m}$.

Fig. $16 \mathrm{Mu}$ opioid receptors co-localize with CGRP or somatostatin in the LHsf. General view of the LHsf (a). Co-localization of mu-mCherry with CGRP (b-d) or somatostatin (e-g) labeling in the LHsf. Nuclei are labeled with DAPI. Scale bars: $a=100 \mu \mathrm{m} ; \mathrm{b}-\mathrm{d}=20 \mu \mathrm{m}$; e-g= $10 \mu \mathrm{m}$.

Fig. 17 The LS-LHsf projection involves neurons expressing mu opioid receptors. Following BDA injection into the LS, BDA anterograde labeling (magenta, b,c) is visible close to LHsf neurons expressing the mu opioid receptor (red, a,c). Following CTB injection in the LHsf, retrogradely labeled neurons (magenta) in the LS are observed among presynaptic enkephalinergic terminals (green) (d; general view). high magnification in the LS shows that neurons expressing the mu opioid receptor (red, e,h) contain CTB (white, f,h) and are surrounded by enkephalinergic terminals (green, g,h). Nuclei are labeled with DAPI. Scale bars: a-c $=10 \mu \mathrm{m} ; \mathrm{d}=100 \mu \mathrm{m} ; \mathrm{e}-\mathrm{h}=10 \mu \mathrm{m}$.

Fig. 18 The central gray projects onto neurons expressing the mu opioid receptor in the LHsf. After BDA injection into the CGA (a), BDA+ axons are observed in low density in LHsf (b). At high resolution, labeled terminals (green, d,e) in the LHsf are visible close to neurons expressing mu opioid receptors (mu-mCherry) (red, c,e). Nuclei are labeled with DAPI. Scale bar: a,b=200 $\mu \mathrm{m} ; \mathrm{c}-\mathrm{e}=10 \mu \mathrm{m}$.

Fig. 19 Summary of the LHsf connectivity. The main inputs (a) and outputs (b) of the LHsf are summarized on a sagittal section of the mouse brain. The density of the connections is indicated by the color code and the size of the structures names. For simplification, the structures with sporadic labeling ("-/+" in table 2) are not included here. See list for abbreviations. 
Table 1: Stereotaxic coordinates for tracers injections.

\begin{tabular}{|l|l|l|l|}
\hline & AP from bregma & DV & ML \\
\hline LHsf & $-0.9 \mathrm{~mm}$ & $-5.25 \mathrm{~mm}$ & $-1 \mathrm{~mm}$ \\
\hline LS & $+0.8 \mathrm{~mm}$ & $-2.7 \mathrm{~mm}$ & $-0.6 \mathrm{~mm}$ \\
\hline $\begin{array}{l}\text { Central gray/nucleus } \\
\text { incertus }\end{array}$ & -5.4 & $-3.4 \mathrm{~mm}$ & $-0.1 \mathrm{~mm}$ \\
\hline
\end{tabular}




\section{Cerebral cortex}

Insular, agranular (AI)

Cingulate

A32

A25

$\mathrm{A} 24 \mathrm{a}$

$\mathrm{A} 24 \mathrm{~b}$

Medial orbital (MO)

Ventral orbital (VO)

Piriform (Pir)

$-/+$

\section{Basal forebrain}

Tenia tecta, dorsal (DTT)

Septal region

Lateral septal N, dorsal (LSD)

Lateral septal N, intermediate (LSI)

Lateral septal N, ventral (LSV)

Septohypothalamic N (SHy)

Medial septal N (MS)

$\mathrm{N}$ of the vertical limb of the diagonal band (VDB)

\section{Basal Ganglia}

Nucleus accumbens, core (AcbC)

Nucleus accumbens, shell (AcbSh)

Ventral pallidum (VP)

\section{Amygdala and extended amygdala}

Anterior amygdaloid area (AAA)

Anterior cortical N (ACo)

Posterior cortical N (PLCo/PMCo)

Amygdalohippocampal area, anterior (AHiA)

Basolateral N, anterior (BLA)

Basolateral N, posterior (BLP)

Basomedial N, anterior (BMA)

Basomedial N, posterior (BMP)

Lateral $\mathrm{N}(\mathrm{La})$

Intercalated N (I)

Sublenticular extended amygdala (EA)

Medial N (MeA)

anterodorsal (MeAD)

anteroventral (MeAV)

posterodorsal (MePD)

posteroventral (MePV)

Bed nucleus of the stria terminalis (BST)

intraamygdaloid division (BSTIA)

lateral division, posterior part (BSTLP)

medial division, anterior part (BSTMA)
$-/+$

$+$

$++$

$-/+$

$+$

$+$

$-/+$

$+$

$+$

$-/+$

$++$

$-1+$

$++++$

$+/++$

$+/++$

$++++$

$++/++$

$+$

$-$

$++/+++$

$-/+$

$-/+$

+ ++

$+/++$

$-/+$

$\begin{array}{ll}-/+ & -/+\end{array}$

$-/+$

$-/+$

$+$

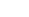

$++$

$+$

$+$

$-/+$

$-$

$-/+$

$-/+$

-

-

$+$

$-/+$

$-$

-

$+/++$

$+$

$+/++$

$-/+$

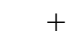

++ +++

$++$

$+$

$+/++$

$+$

- $-1+$

$+$

$-1+$ 


\begin{tabular}{|c|c|c|}
\hline medial division, ventral part (BSTMV) & + & $-/+$ \\
\hline medial division, posterior part (BSTMP) & $+/++$ & + \\
\hline \multicolumn{3}{|l|}{ Dorsal Thalamus } \\
\hline Mediodorsal N (MD) & - & $-/+$ \\
\hline Centromedial $\mathrm{N}(\mathrm{CM})$ & - & $-1+$ \\
\hline $\begin{array}{l}\text { Posterior triangular } \mathrm{N}(\mathrm{PoT}) / \text { Posterior intralaminar } \mathrm{N} \\
\text { (PIL) }\end{array}$ & + & - \\
\hline Paratenial N (PT) & - & $+/++$ \\
\hline Paraventricular N (PV) & - & + \\
\hline Reuniens N (Re) & - & $+/++$ \\
\hline \multicolumn{3}{|l|}{ Ventral thalamus } \\
\hline Zona incerta (ZI) & $-/+$ & + \\
\hline \multicolumn{3}{|l|}{ Epithalamus } \\
\hline Lateral habenular $\mathrm{N}(\mathrm{LHb})$ & - & $+++/++++$ \\
\hline \multicolumn{3}{|l|}{ Hypothalamus } \\
\hline \multicolumn{3}{|l|}{ Periventricular zone } \\
\hline Median preoptic $\mathrm{N}(\mathrm{MnPo})$ & $-/+$ & - \\
\hline Paraventricular N (Pa) & ++ & $-/+$ \\
\hline Supraoptic N (SO) & ++ & - \\
\hline Striohypothalamic N (StHy) & $-/+$ & - \\
\hline \multicolumn{3}{|l|}{ Medial zone } \\
\hline Anterior area $(\mathrm{AH})$ & $-/+$ & $+/++$ \\
\hline Medial preoptic area (MPA) & $+/++$ & + \\
\hline Medial preoptic N (MPO) & + & - \\
\hline Dorsomedial N (DM) & $-/+$ & $-/+$ \\
\hline Ventromedial $\mathrm{N}(\mathrm{VMH})$ & +++ & $++/+++$ \\
\hline Premamillary N, dorsal (PMD) & +++ & +++ \\
\hline Premamillary N, ventral (PMV) & $-/+$ & - \\
\hline Retromamillary N (RM) & $-/+$ & + \\
\hline Posterior area $(\mathrm{PH})$ & + & + \\
\hline Medial mamillary N, lateral and medial parts (MM, ML) & - & $-/+$ \\
\hline \multicolumn{3}{|l|}{ Lateral zone } \\
\hline Lateral preoptic area (LPO) & + & $+/++$ \\
\hline Lateral area $(\mathrm{LH})$ & nd & nd \\
\hline \multicolumn{3}{|l|}{ Brainstem } \\
\hline \multicolumn{3}{|l|}{ Periaqueductal gray (PAG) } \\
\hline rostral (r PAG) & $+/++$ & ++ \\
\hline dorsomedial (dmPAG) & $-/+$ & + \\
\hline dorsolateral (dlPAG) & $+/++$ & $-1+$ \\
\hline lateral (IPAG) & + & $+/++$ \\
\hline ventrolateral (vlPAG) & $-/+$ & + \\
\hline \multicolumn{3}{|l|}{ Parabrachial area } \\
\hline Lateral parabrachial N, superior part (LBPS) & + & - \\
\hline Lateral parabrachial N, central part (LPBC) & ++ & $-/+$ \\
\hline Lateral parabrachial N, dorsal part (LPBD) & $-/+$ & - \\
\hline Median / paramedian raphe N (MnR/PMnR) & + & + \\
\hline
\end{tabular}




\begin{tabular}{lcc}
\hline Rostral linear N (RLi) & $-/+$ & $-/+$ \\
Dorsal raphe N (DR) & $-/+$ & $+/++$ \\
Mesencephalic reticular area (mRt) & $-/+$ & $-/+$ \\
Cuneiform N (CnF) & + & $-/+$ \\
Anterior tegmental N (ATg) & - & + \\
Laterodorsal tegmental N (LDTg) & $-/+$ & $-/+$ \\
Pedunculotegmental tegmental N (PTg) & $-/+$ & - \\
Ventral tegmental area (VTA) & $-/+$ & $+/++$ \\
Substantia nigra, pars compacta (SNC) & - & $-/+$ \\
Medial paralemniscal N (MPL) & $++/+++$ & - \\
Locus coeruleus (LC)/ Barrington's N (Bar) & $-/+$ & $-/+$ \\
Central gray alpha/beta parts (CGA/CGB) & + & $-/+$ \\
\hline
\end{tabular}

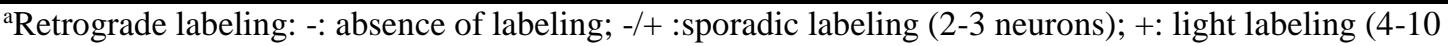
neurons); ++: moderate labeling (11-30 neurons); +++: dense labeling (30-50 neurons); ++++: very dense labeling ( $>50$ neurons); nd: non determined

${ }^{\mathrm{b}}$ Anterograde labeling: : -: absence of labeling; + low labeling; ++: moderate labeling; +++: dense labeling; ++++ : very dense labeling; nd: non determined 

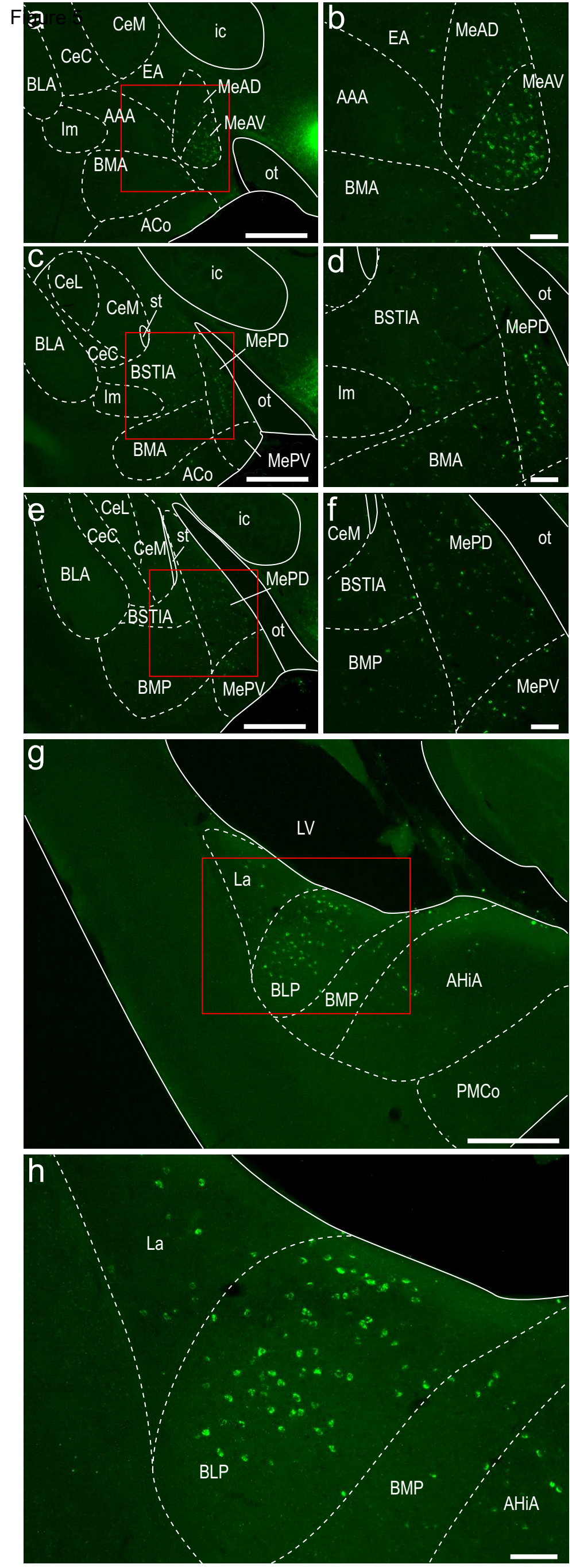

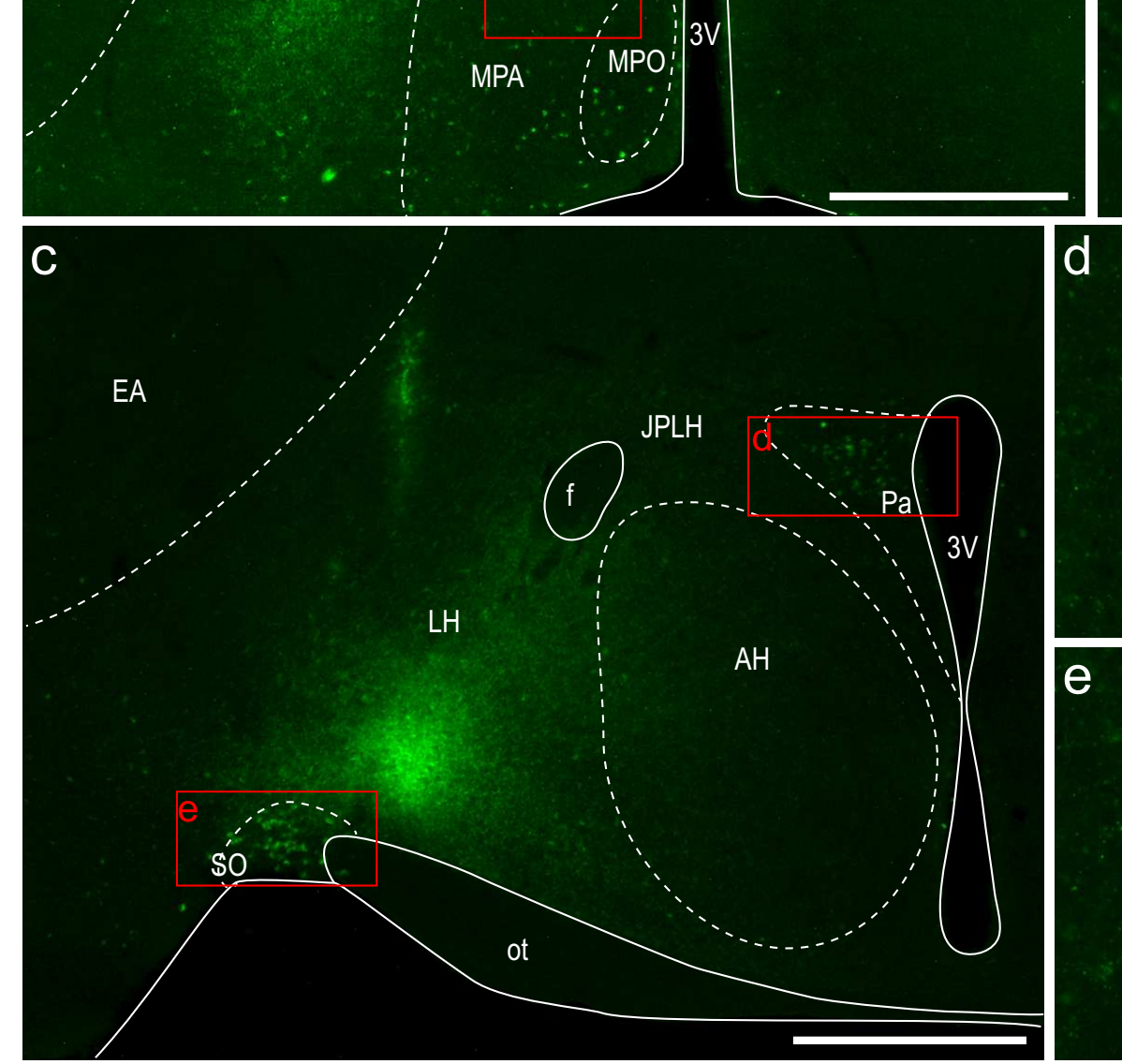

-
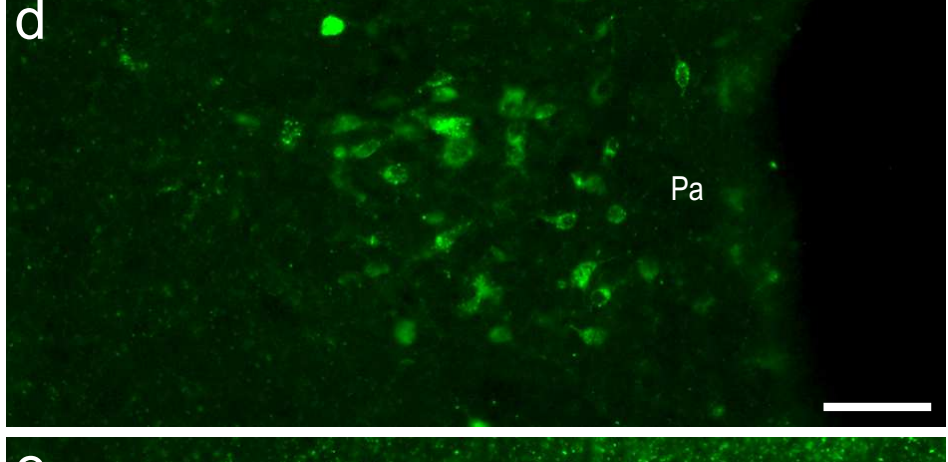

e 
$\mathrm{F} 9$

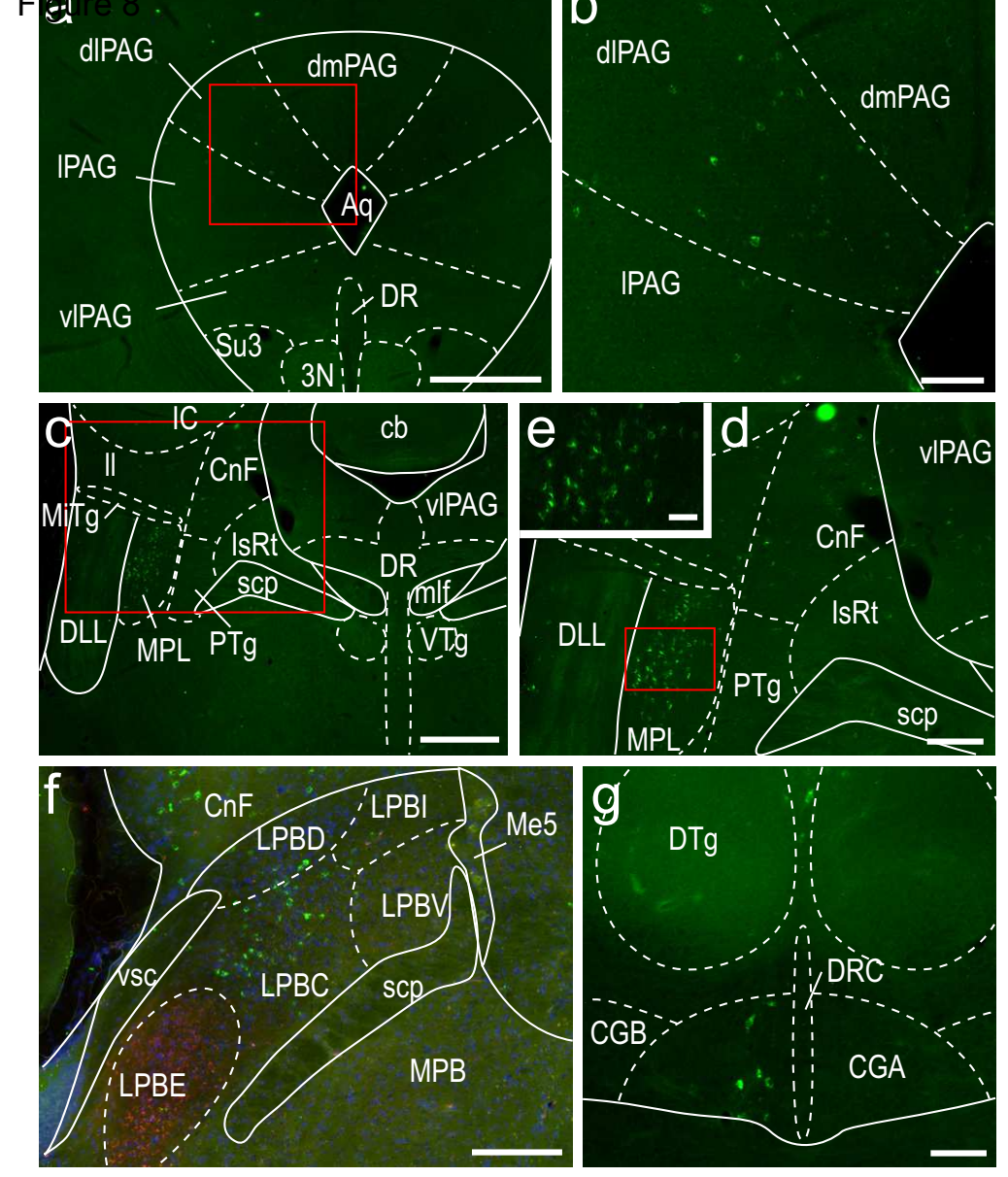




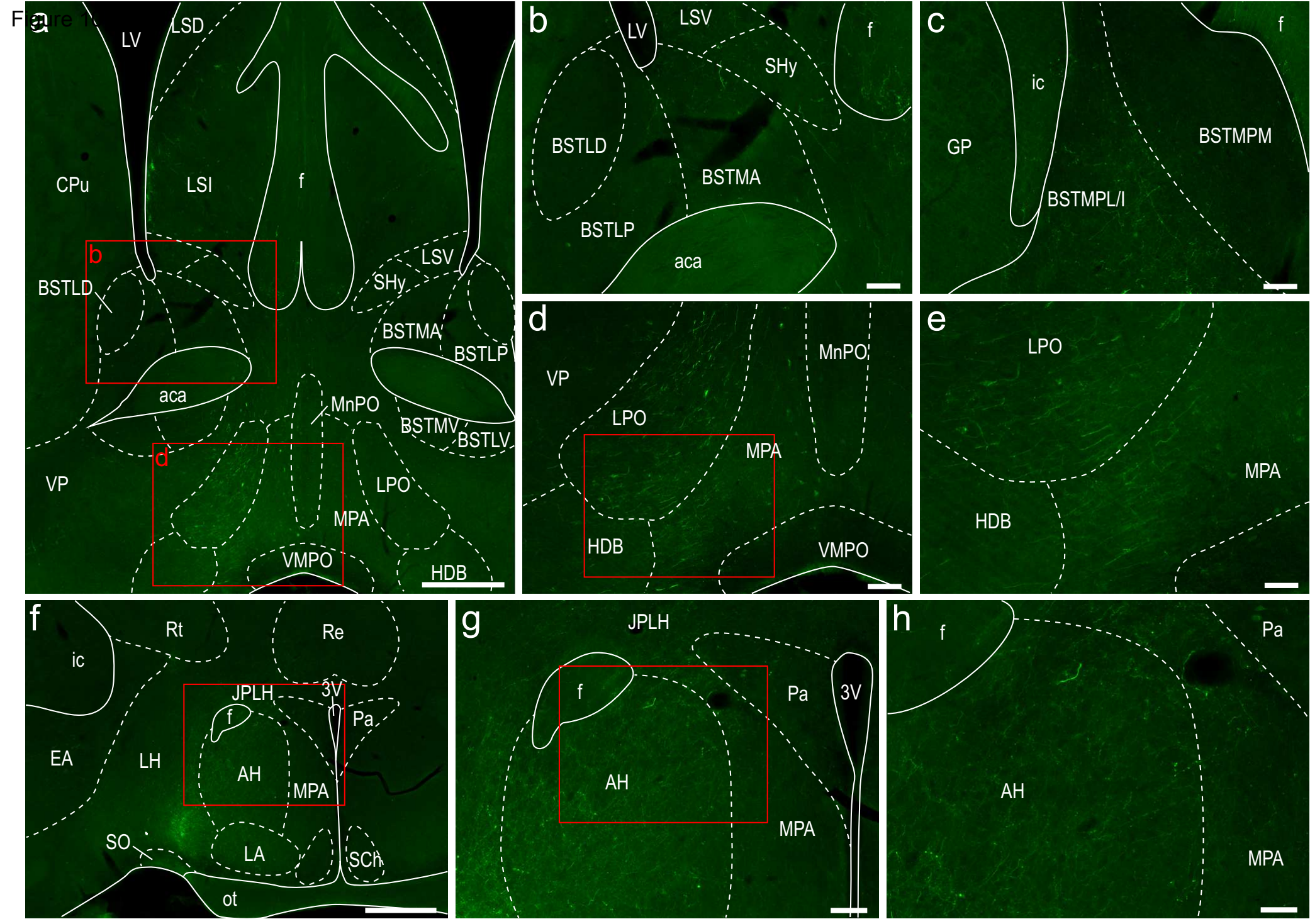



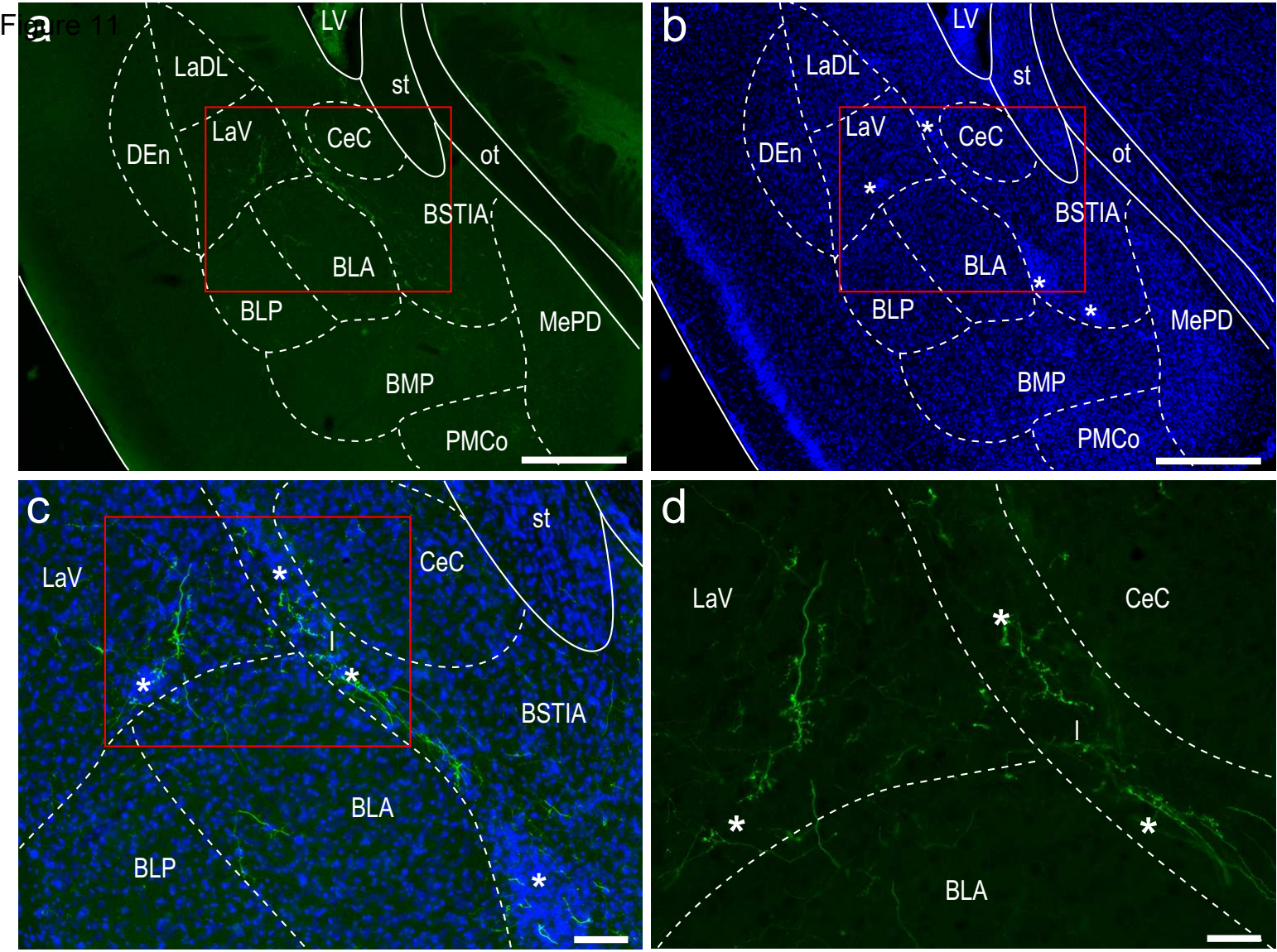

e

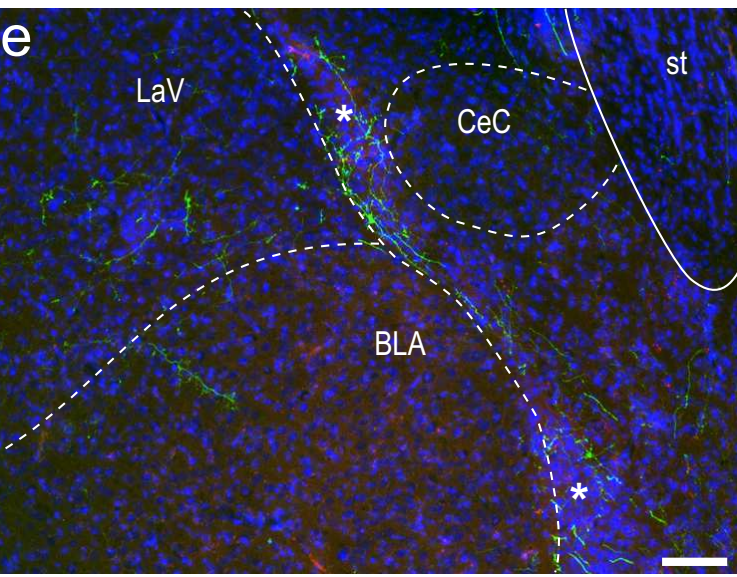

9
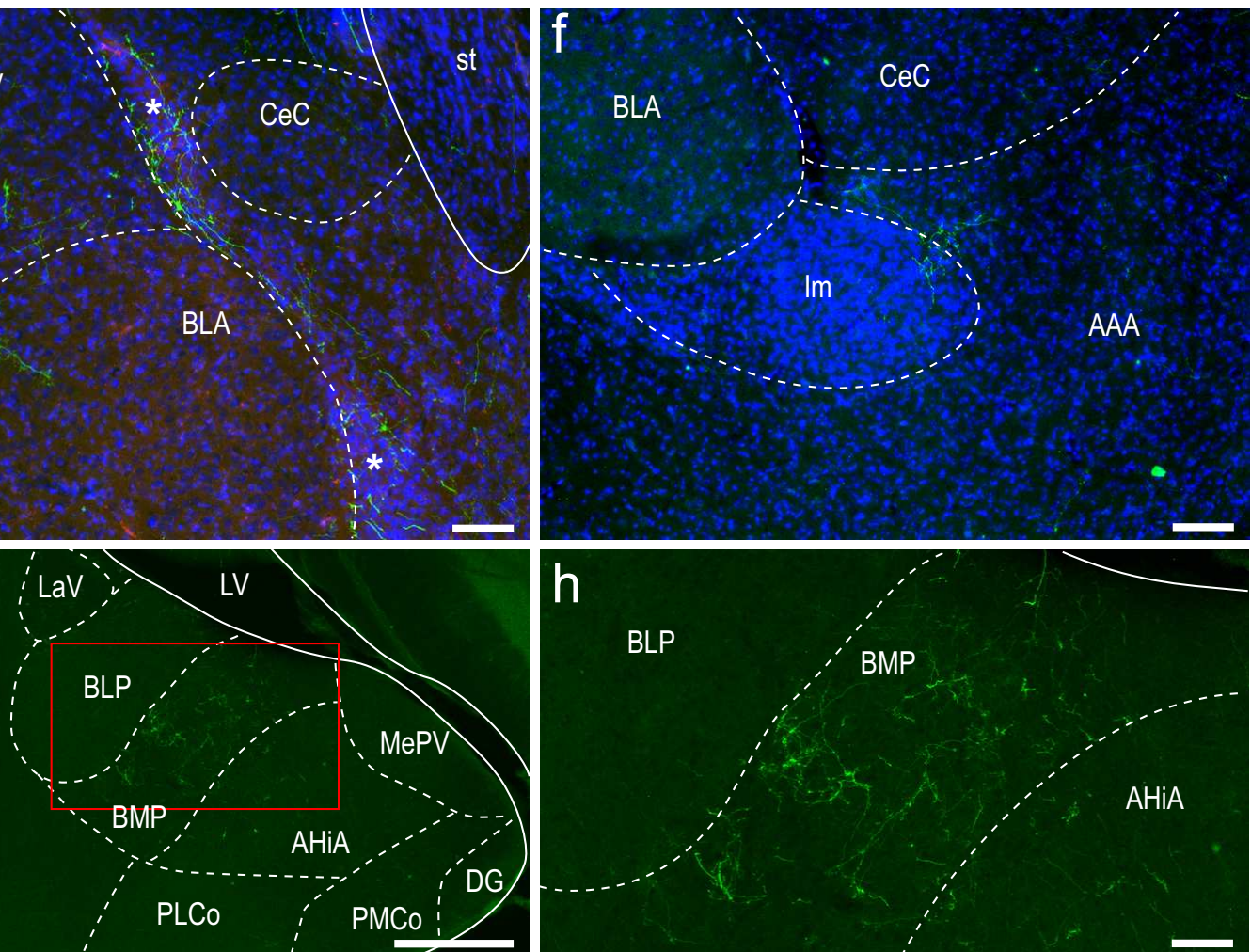

h

AHIA

\section{BMP}

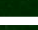




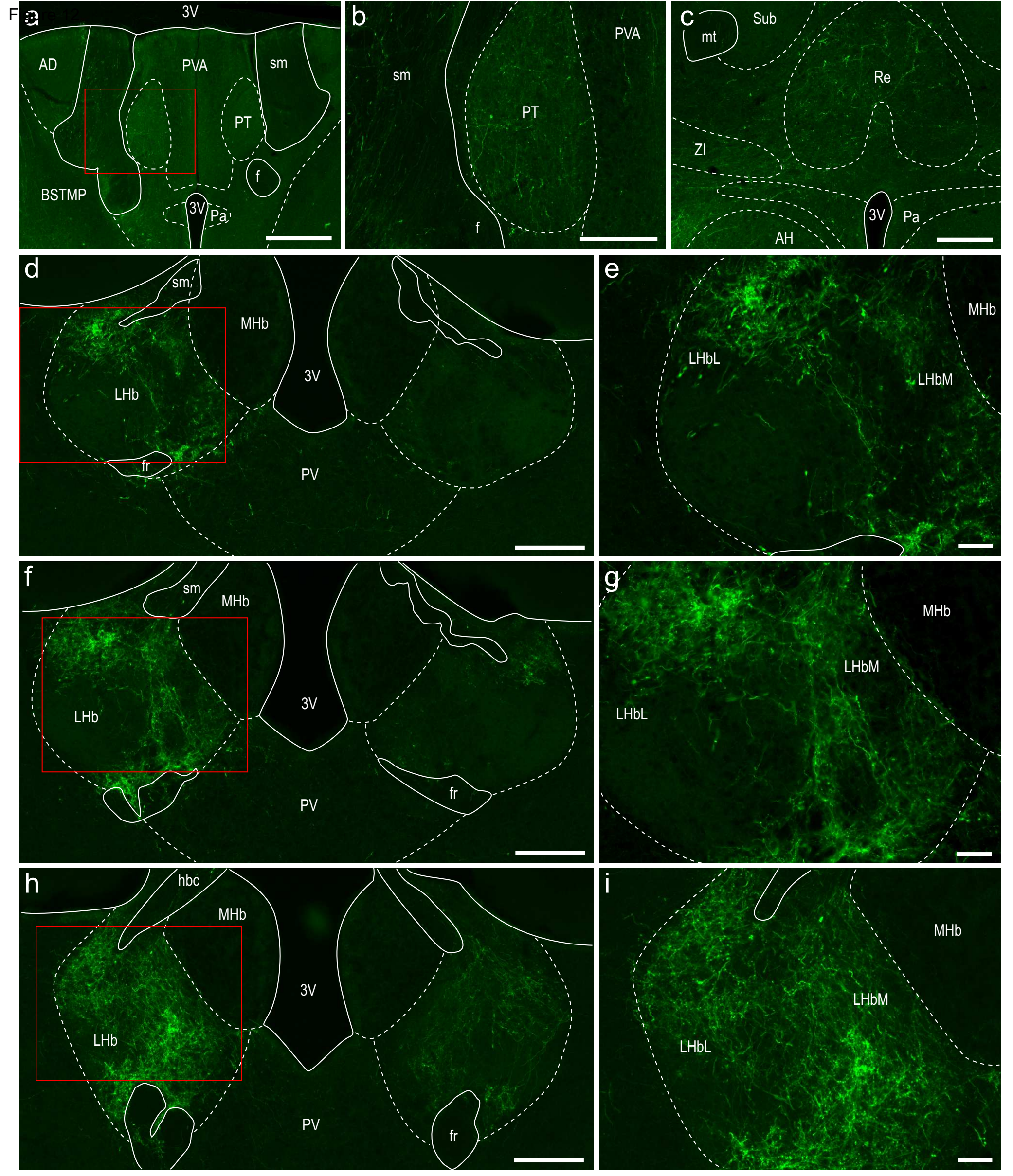




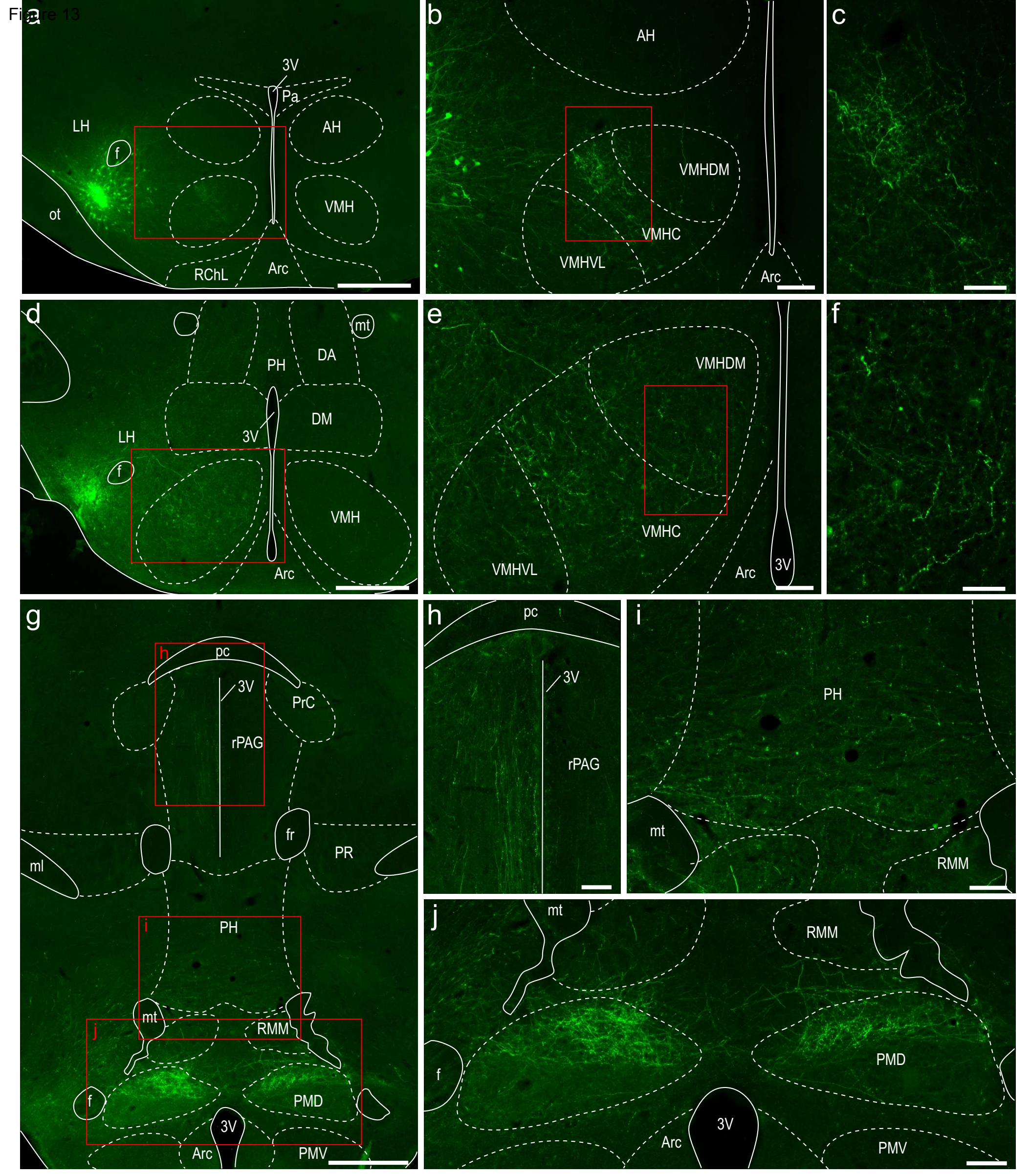



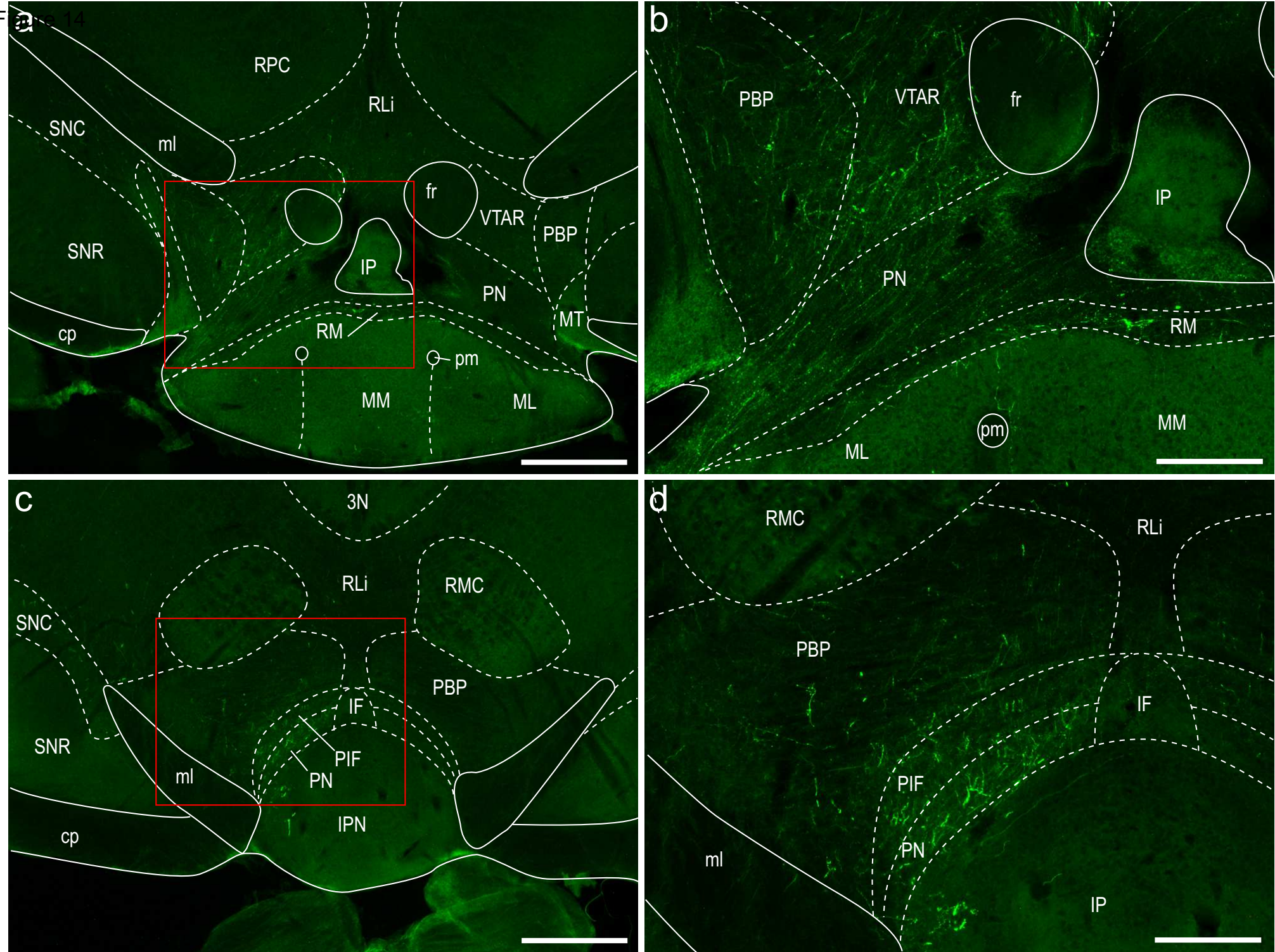


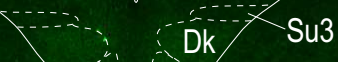
mlf MA3

IPAG
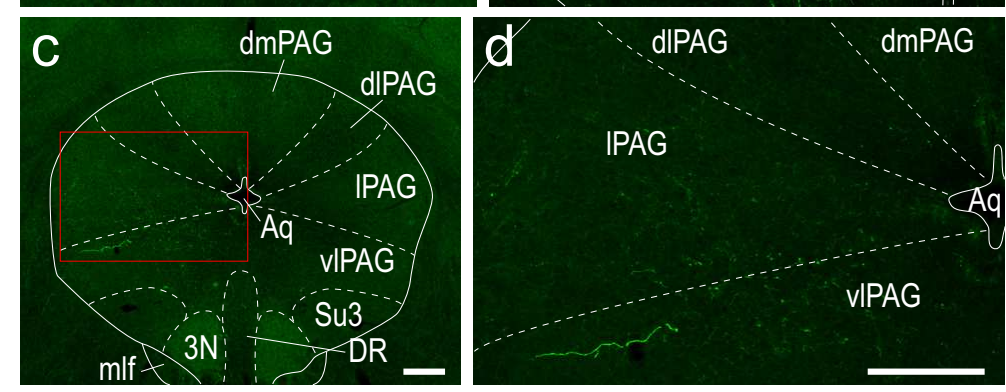

e

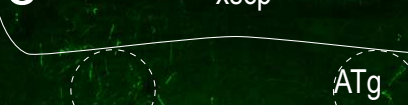

ÁTg.

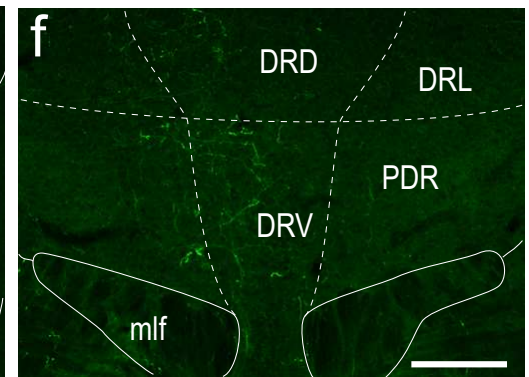

$\mathrm{MnR}$

PMnR

*

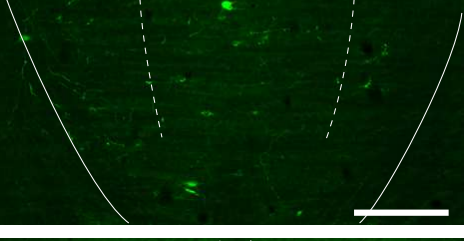

g

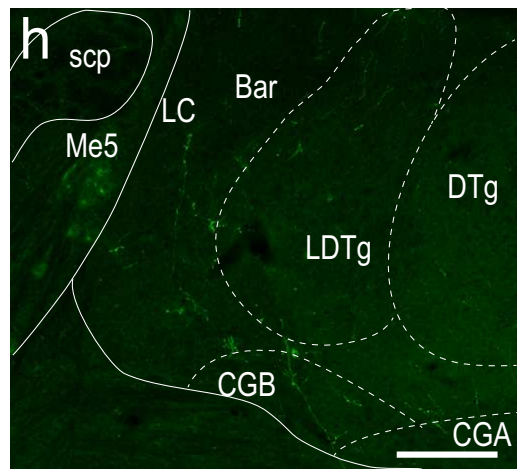

CGA 


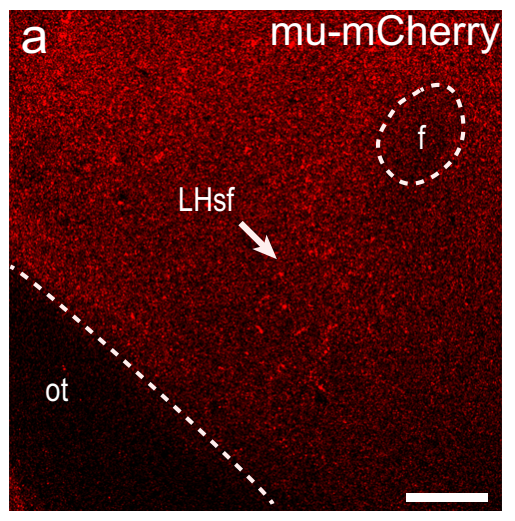

e

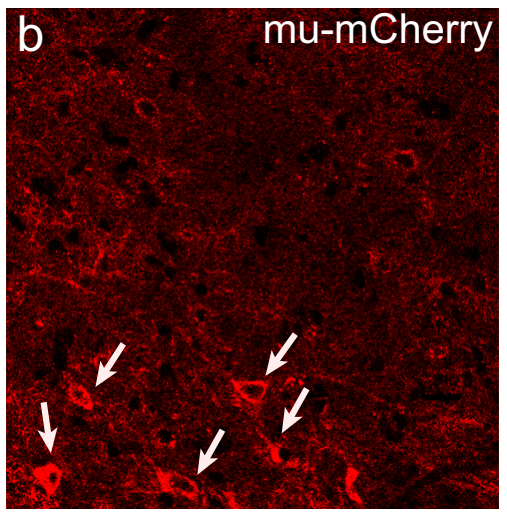

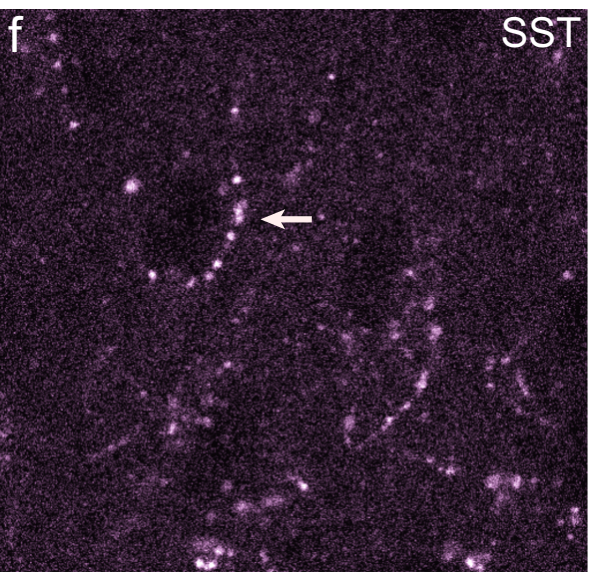
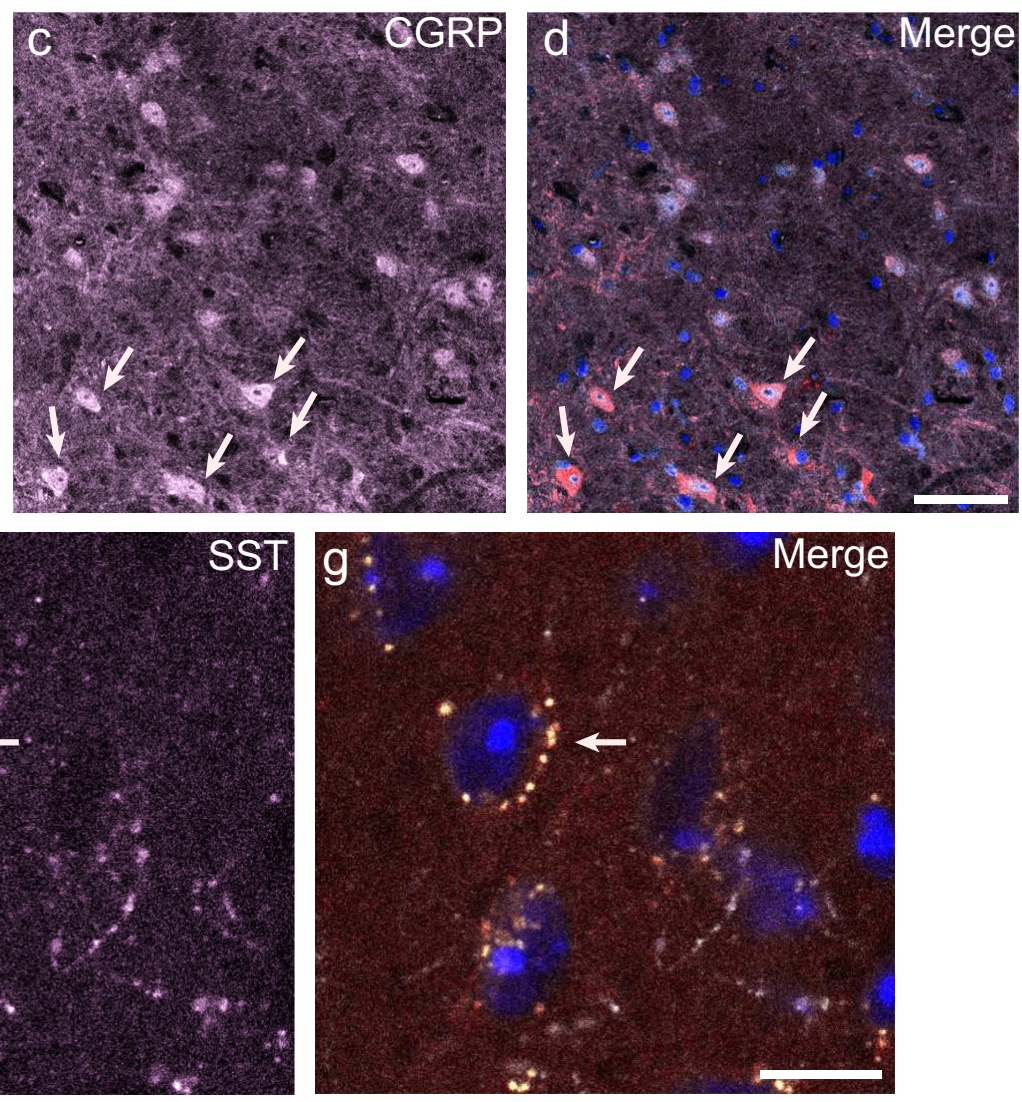

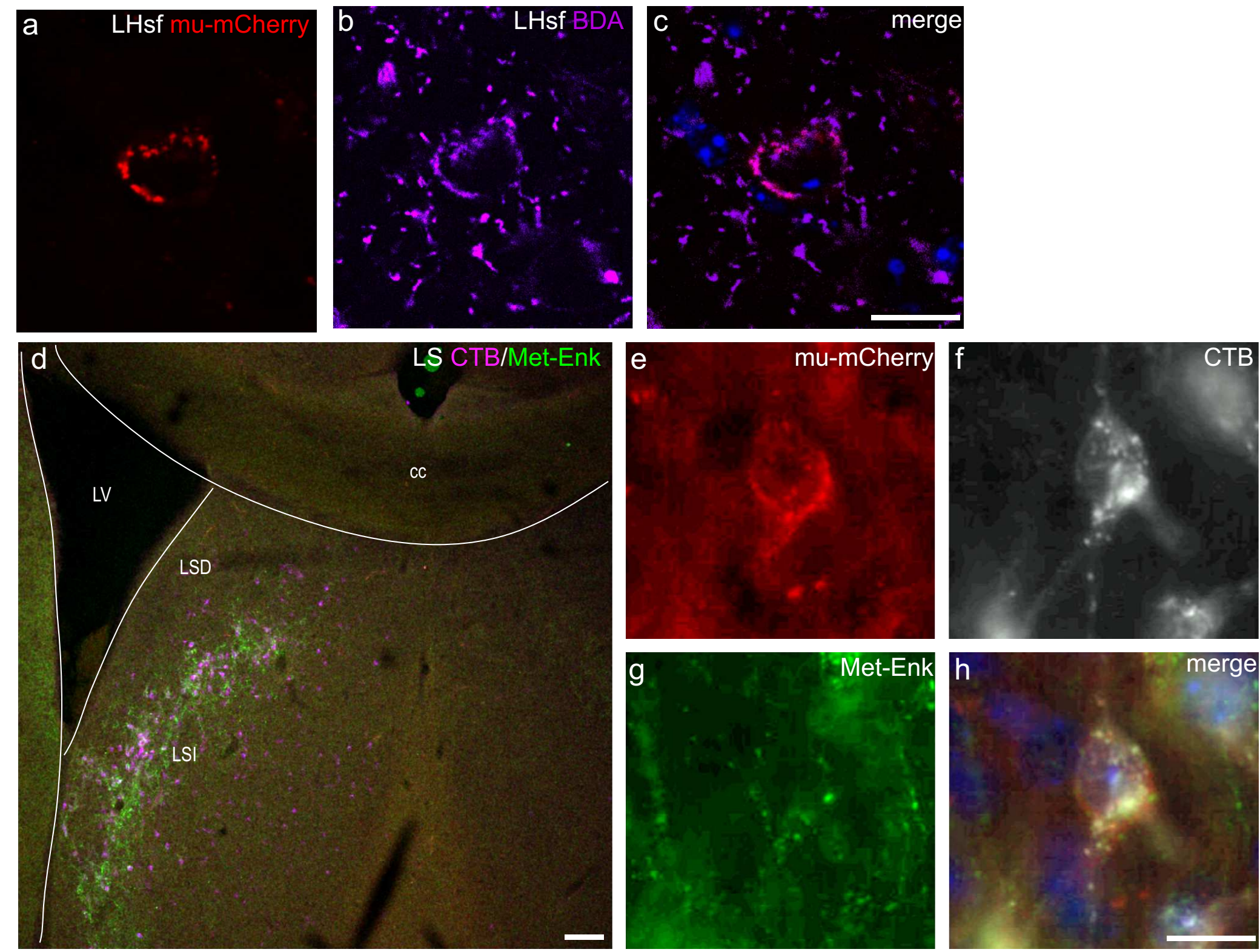

g

Met-Enk

merge
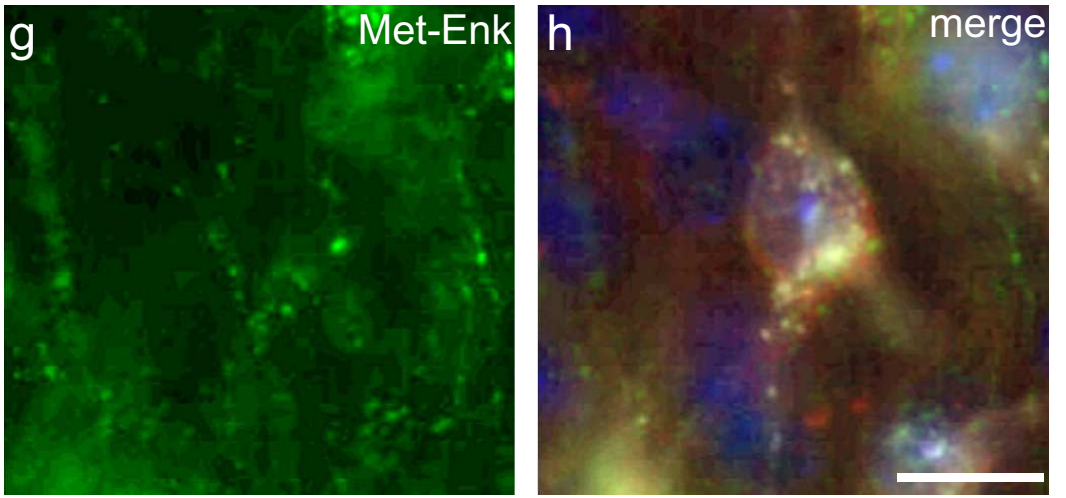

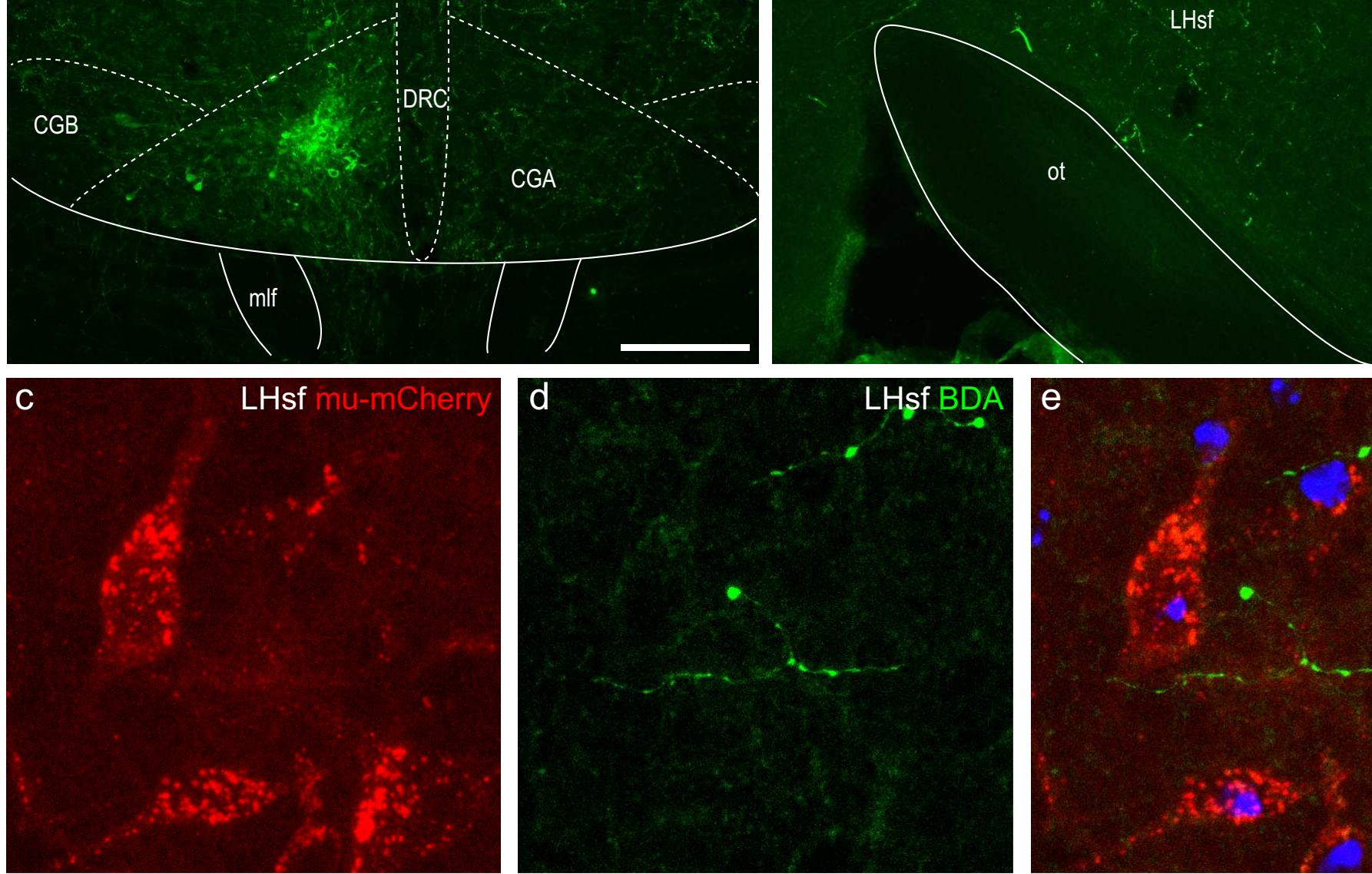

e

merge

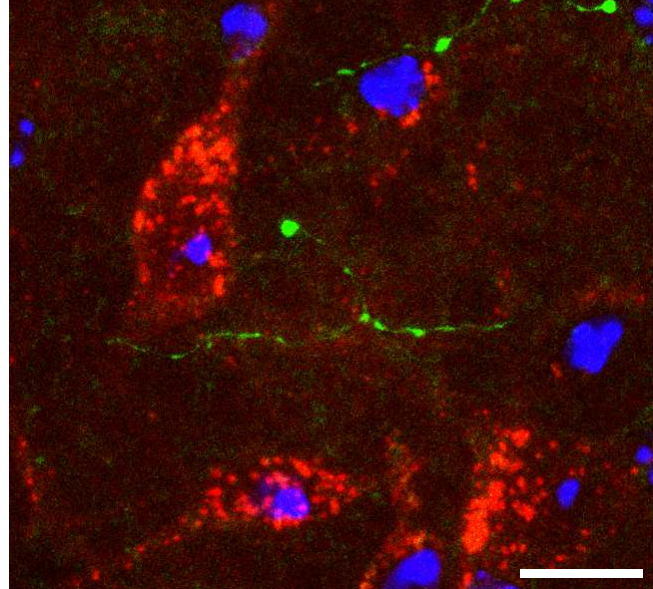


Dear Editor,

Please find attached the revision of our manuscript entitled " Connections of the mouse subfornical region of the lateral hypothalamus (LHsf)" by Müzeyyen Ugur, Stéphane Doridot, Susanne E la Fleur, Pierre Veinante and Dominique Massotte. (Manuscript Number: BSAF-D-20-00052R2).

We thank the reviewers for their useful comments that helped us strengthening our manuscript. We hope that this revision of our manuscript constructively answered the reviewer's comments, and that it may now be positively considered for publication in Brain Structure \& Function.

For the authors,

Pr. Pierre Veinante 